\title{
Nerves and classifying spaces for bicategories
}

\author{
Pilar CARRASCO \\ ANTONIO M CEgARRA \\ ANTONIO R GARZÓN
}

\begin{abstract}
This paper explores the relationship amongst the various simplicial and pseudosimplicial objects characteristically associated to any bicategory $\mathcal{C}$. It proves the fact that the geometric realizations of all of these possible candidate "nerves of $\mathcal{C}$ " are homotopy equivalent. Any one of these realizations could therefore be taken as the classifying space $\mathrm{BC}$ of the bicategory. Its other major result proves a direct extension of Thomason's "Homotopy Colimit Theorem" to bicategories: When the homotopy colimit construction is carried out on a diagram of spaces obtained by applying the classifying space functor to a diagram of bicategories, the resulting space has the homotopy type of a certain bicategory, called the "Grothendieck construction on the diagram". Our results provide coherence for all reasonable extensions to bicategories of Quillen's definition of the "classifying space" of a category as the geometric realization of the category's Grothendieck nerve, and they are applied to monoidal (tensor) categories through the elemental "delooping" construction.
\end{abstract}

18D05; 55U40

\section{Introduction and summary}

Higher-dimensional categories provide a suitable setting for the treatment of an extensive list of subjects with recognized mathematical interest. The construction of nerves and classifying spaces of higher categorical structures, and bicategories in particular, discovers ways to transport categorical coherence to homotopical coherence and it has shown its relevance as a tool in algebraic topology, algebraic geometry, algebraic $K$-theory, string theory, conformal field theory and in the study of geometric structures on low-dimensional manifolds.

This paper explores the relationship amongst the various simplicial and pseudosimplicial objects that have been (or might reasonably be) functorially and characteristically associated to any bicategory $\mathcal{C}$. It outlines and proves in detail the far from obvious fact that the geometric realizations of all of these possible candidate "nerves of $\mathcal{C}$ " are homotopy equivalent. Any one of these realizations could therefore be taken as the 
classifying space $\mathrm{BC}$ of the bicategory. Since this result quite reasonably extends, to bicategories, Quillen's definition [35] of the "classifying space" of a category such as $\mathrm{BNC}$, the geometric realization of the category's Grothendieck nerve, and given that monoidal (tensor) categories may be identified with bicategories having a single object, the paper may quite possibly be of special interest to $K$-theorists as well as to researchers interested in homotopy theory of higher categorical structures. Moreover, the other nonelementary result of the paper gives and nontrivially proves a direct extension of Thomason's "Homotopy Colimit Theorem" [43] to bicategories: When the homotopy colimit construction is carried out on a diagram of spaces obtained by applying the classifying space functor to a diagram of bicategories, the resulting space has the homotopy type of a certain bicategory called the "Grothendieck construction on the diagram". Notice that, even in the case where the diagram is of monoidal categories and monoidal functors, the Grothendieck construction on it turns out to be a genuine bicategory. Hence, the reader interested in the study of classifying spaces of monoidal categories can find in the above fact a good reason to also be interested in the study of classifying spaces of bicategories.

There is a miscellaneous collection of ten different "nerves" for a bicategory $\mathcal{C}$ discussed in the paper, each with a particular functorial property. All these nerves occur in a commutative diagram

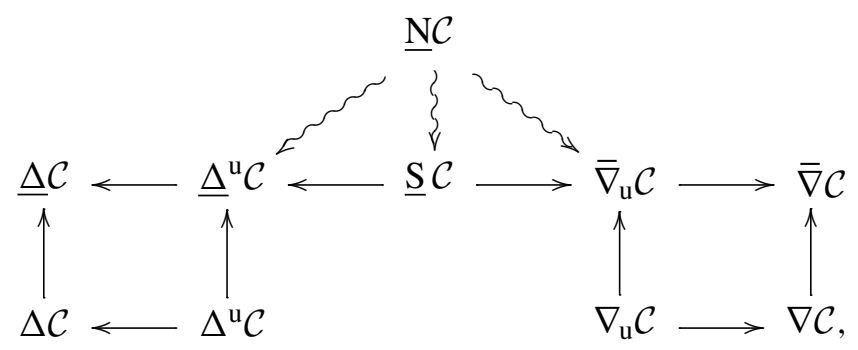

in which the arrows written as $\rightarrow$ denote simplicial maps (those in the bottom row) or simplicial functors (all the others), whereas those written as $\rightsquigarrow$ are pseudosimplicial functors.

In the diagram, $\underline{N C}: \Delta^{\text {op }} \rightsquigarrow$ Cat is the pseudosimplicial category whose category of $p$-simplices is

$$
\underline{\mathrm{N}}_{p}=\bigsqcup_{\left(x_{0}, \ldots, x_{p}\right) \in \mathrm{Ob} \mathcal{C}^{p+1}} \mathcal{C}\left(x_{1}, x_{0}\right) \times \mathcal{C}\left(x_{2}, x_{1}\right) \times \cdots \times \mathcal{C}\left(x_{p}, x_{p-1}\right),
$$

where $\mathcal{C}(x, y)$ denotes the hom-category of the bicategory $\mathcal{C}$ at a pair of objects $(x, y)$. The face and degeneracy functors are defined in the standard way by using the horizontal composition and the identity morphisms of the bicategory, and the 
natural isomorphisms $d_{i} d_{j} \cong d_{j-1} d_{i}$, etc, being given from the associativity and unit constraints of the bicategory. Therefore, when a category $\mathcal{C}$ is considered as a discrete bicategory, that is, where the deformations (2-cells) are all identities, then $\underline{N} \mathcal{C}=\mathrm{NC}$, the usual Grothendieck's nerve of the category. This Cat-valued "pseudosimplicial nerve" of a bicategory is explained in some detail in Section 3 of the paper, and its realization is taken as the "classifying space" $\mathrm{BC}$ of the bicategory. Since the horizontal composition involved is in general neither strictly associative nor unitary, $\underline{\mathrm{NC}}$ is not a simplicial category. Consequently, we are, unfortunately, forced to deal with defining the geometric realization of what is not simplicial but only "simplicial up to isomorphisms". Indeed, as we review in the preliminary Section 2, this has been done by Segal, Street and Thomason using some of Grothendieck's methods, but the process is quite indirect and the $\mathrm{CW}$-complex $\mathrm{BC}$ thus obtained has little apparent intuitive connection with the cells of the original bicategory $\mathcal{C}$.

However, the rest of the nerves in the diagram above do not have these simplicial defects caused by the lack of associativity or unitary properties of the horizontal composition in the bicategory. To refer briefly to some of them here, say that, for example, $\Delta^{\mathrm{u}} \mathcal{C}: \Delta^{\mathrm{op}} \rightarrow$ Set and $\nabla_{\mathrm{u}} \mathcal{C}: \Delta^{\mathrm{op}} \rightarrow$ Set are the nerves of the bicategory introduced by Street in [41] and Duskin in [15]. These are genuine single simplicial sets, termed here the "unitary geometric nerves" of the bicategory. As observed by Duskin (see also Gurski [23], for an interesting new approach), both $\Delta^{\mathrm{u}} \mathcal{C}$ and $\nabla_{\mathrm{u}} \mathcal{C}$ completely encode all the structure of the bicategory and, furthermore, there is a pleasing geometrical description of their simplices: a $p$-simplex of $\Delta^{\mathrm{u}} \mathcal{C}$ (resp. of $\nabla_{\mathrm{u}} \mathcal{C}$ ) is geometrically represented by a diagram in $\mathcal{C}$ with the shape of the 2 -skeleton of an oriented affine standard $p$-simplex, whose faces are triangles
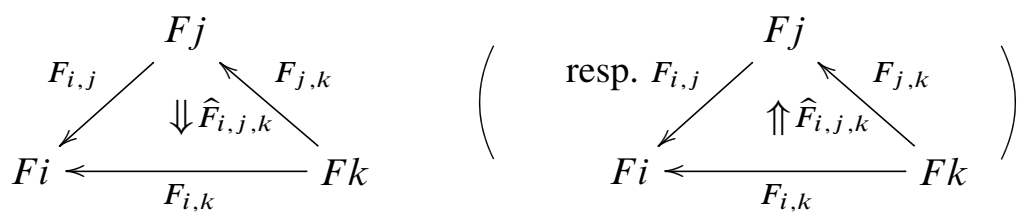

with objects ( 0 -cells) $F i$ placed on the vertices, $0 \leq i \leq p$, morphisms (1-cells) $F_{i, j}: F j \rightarrow F i$ on the edges, $0 \leq i<j \leq p$, and $\widehat{F}_{i, j, k}: F_{i, j} \circ F_{j, k} \Rightarrow F_{i, k}$ (resp. $\widehat{F}_{i, j, k}: F_{i, k} \Rightarrow F_{i, j} \circ F_{j, k}$ ) deformations (2-cells) in $\mathcal{C}$, for $0 \leq i<j<k \leq p$.

The other two simplicial sets in the diagram, $\Delta \mathcal{C}: \Delta^{\text {op }} \rightarrow$ Set and $\nabla \mathcal{C}: \Delta^{\text {op }} \rightarrow$ Set, here termed "geometric nerves" of $\mathcal{C}$, are respective nonnormalized versions of the above unitary ones by Street and Duskin. And the remaining five "nerves" in the diagram associate to every bicategory $\mathcal{C}$ simplicial objects in Cat: Those called its "unitary categorical geometric nerves", which are those denoted by $\underline{\Delta}^{\mathrm{u}} \mathcal{C}$ and $\bar{\nabla}_{\mathrm{u}} \mathcal{C}$, its "categorical geometric nerves", denoted by $\underline{\Delta C}$ and $\bar{\nabla} \mathcal{C}$, and its "Segal nerve", written 
as $\underline{S} \mathcal{C}$, respectively. As we will prove, all these are "special" simplicial categories, in the sense that the Segal projection maps on them induce homotopy equivalences on classifying spaces $[37 ; 43]$. Furthermore, the latter, $\underline{S} \mathcal{C}$, is a weak 2 -category in the sense of Tamsamani [42] and Simpson [38], that is, the Segal projection maps on it are surjective equivalences of categories as observed by Lack and Paoli in [29] (where $\underline{S} \mathcal{C}$ is called the "2-nerve of $\mathcal{C}$ ", but this may be confusing terminology since, for example, the unitary geometric nerve $\underline{\Delta}^{\mathrm{u}} \mathcal{C}$ of a 2 -category $\mathcal{C}$ is also called the "2-nerve of $\mathcal{C}$ " in Worytkiewicz et al [46]).

Our first major result with this work states and proves that:

Theorem 6.1 For any bicategory $\mathcal{C}$, all the continuous maps in the diagram

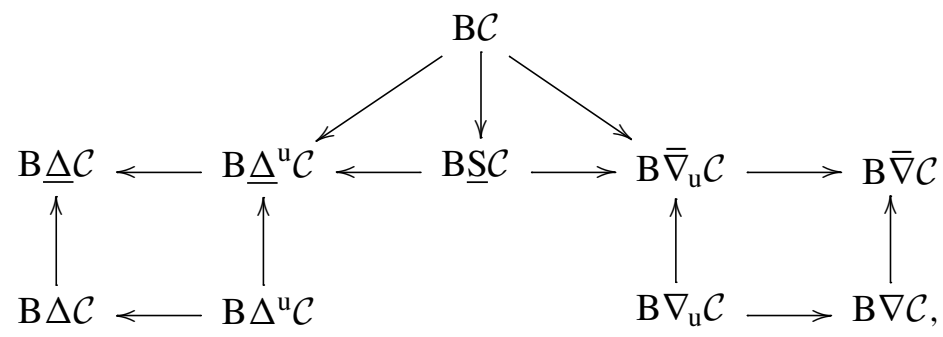

induced by (A) on classifying spaces, are homotopy equivalences.

Many properties of the classifying space construction for bicategories, $\mathcal{C} \mapsto \mathrm{BC}$, may be easier to establish depending on the nerve used for realizations. For example, in what is our second main result, we deal with the homotopy theory of diagrams of bicategories and homomorphisms $\mathcal{C}: I^{\mathrm{op}} \rightarrow$ Bicat. Following Grothendieck [21], we show the existence of a "bicategorical Grothendieck construction" $\int_{I} \mathcal{C}$, that suitably assembles all bicategories $\mathcal{C}_{i}, i \in \mathrm{Ob} I$, and whose classifying space $\mathrm{B} \int_{I} \mathcal{C}$ can be thought as the homotopy colimit of the diagram of spaces $i \mapsto \mathrm{B} \mathcal{C}_{i}$, as we prove by using geometric nerves for realizing classifying spaces of bicategories. More precisely, we prove the following:

Theorem 7.3 Suppose a category $I$ is given. For every functor $\mathcal{C}: I^{\mathrm{op}} \rightarrow$ Bicat, there exists a natural weak homotopy equivalence of simplicial sets

$$
\eta: \operatorname{hocolim}_{I} \Delta \mathcal{C} \longrightarrow \Delta \int_{I} \mathcal{C}
$$

where $\Delta \mathcal{C}: I^{\mathrm{op}} \rightarrow$ Simpl.Set is the diagram of simplicial sets obtained by composing $\mathcal{C}$ with the geometric nerve of bicategories functor. 
We should stress that, regarding any category as a discrete bicategory, the above weak equivalence $\eta$ for diagrams of categories $\mathcal{C}: I^{\text {op }} \rightarrow$ Cat just gives the weak equivalence shown by Thomason in his well-known Homotopy Colimit Theorem [43]. However, our proof that $\eta$ is a weak homotopy equivalence follows different lines than Thomason's. The plan of this paper is, briefly, as follows. After this introductory Section 1, the paper is organized in six sections. Section 2 aims to make this paper as self-contained as possible; hence, at the same time as we fix notation and terminology, we also review some necessary aspects and results from the background of simplicial sets, homotopy theory of categories and bicategories. However, the material in Section 2 is quite standard, so the expert reader may skip most of it. In Section 3, we use Jardine's supercoherence theorem [25] to introduce the pseudosimplicial nerve $\underline{\mathrm{NC}}$ of a bicategory and then Thomason's theory on lax-diagrams of categories define its classifying space $\mathrm{BC}$. Sections 4 and 5 are dedicated to describing the remaining nerves of a bicategory discussed in the paper as well as the simplicial maps, functors or pseudofunctors that connect to each other. In Section 6, we include our main result on the homotopy invariance of all the different nerves of a bicategory, and the final Section 7 mainly contains our homotopy colimit theorem for diagrams of bicategories.

Acknowledgements This work was partially supported by DGI of Spain and FEDER (Project: MTM2007-65431); Consejería de Innovacion de J. de Andalucía (P06FQM-1889); MEC de España, "Ingenio Mathematica(i-Math)” No. CSD2006-00032 (consolider-Ingenio 2010).

\section{Preliminaries}

In this preliminary section we review, without any claim to originality, some standard constructions and well-known basic facts mainly concerning nerves and classifying spaces of (small) categories. In Section 2.4, we fix some terminology and notational conventions on bicategories that for some readers may be idiosyncratic.

\subsection{Some facts concerning simplicial sets}

Hereafter, we shall regard each ordered set $[n]=\{0,1, \ldots, n\}$ as the category with exactly one arrow $j \rightarrow i$ if $i \leq j$. Then, a nondecreasing map $[n] \rightarrow[m]$ is the same as a functor, so that we see $\Delta$, the simplicial category of finite ordinal numbers, as a full subcategory of Cat, the category (actually the 2-category) of small categories.

The category of simplicial sets, that is, the category of functors $S: \Delta^{\mathrm{op}} \rightarrow$ Set, where Set is the category of sets, is denoted by Simpl.Set. The simplicial standard $n$-simplex $\Delta[n]=\operatorname{Hom}_{\Delta^{\mathrm{op}}}(-,[n])$ is the obvious representable functor, as usual. 
Recall that the category $\Delta$ is generated by the injections $d^{i}:[n-1] \rightarrow[n]$ (cofaces), $0 \leq$ $i \leq n$, which omit the $i$-th element and the surjections $s^{i}:[n+1] \rightarrow[n]$ (codegeneracies), $0 \leq i \leq n$, which repeat the $i$-th element, subjet to the well-known cosimplicial identities: $d^{j} d^{i}=d^{i} d^{j-1}$ if $i<j$, etc. Thus, in order to define a simplicial object in a category $\mathcal{E}$, say $S: \Delta^{\mathrm{op}} \rightarrow \mathcal{E}$, it suffices to give the objects (of $n$-simplices) $S_{n}$, $n \geq 0$, together with morphisms

$$
\begin{array}{lll}
d_{i}=\left(d^{i}\right)^{*}=S\left(d^{i}\right): S_{n} \rightarrow S_{n-1}, & 0 \leq i \leq n \quad \text { (the face operators) } \\
s_{i}=\left(s^{i}\right)^{*}=S\left(s^{i}\right): S_{n} \rightarrow S_{n+1}, & 0 \leq i \leq n \quad \text { (the degeneracy operators), }
\end{array}
$$

satisfying the well-known basic simplicial identities: $d_{i} d_{j}=d_{j-1} d_{i}$ if $i<j$, etc. A simplicial morphism $f: S \rightarrow S^{\prime}$ is just a natural transformation from $S$ to $S^{\prime}$; it then consists of a family $\left\{f_{n}: S_{n} \rightarrow S_{n}^{\prime}, n \geq 0\right\}$ of arrows in $\mathcal{E}$ that commute with the face and degeneracy operators. If $f, g: S \rightarrow S^{\prime}$ are simplicial morphisms, then a simplicial homotopy $H: f \Rightarrow g$ is a system $\left\{H_{m}: S_{n} \rightarrow S_{n+1}^{\prime}, 0 \leq m \leq n\right\}$ of arrows in $\mathcal{E}$ that satisfies the set of homotopy identities described, for example, in May [31, Definition 5.1].

Segal's geometric realization [36] of a simplicial (compactly generated topological) space $S: \Delta^{\mathrm{op}} \rightarrow$ Top is denoted by B $S$. This construction is functorial and any simplicial homotopy $H: f \Rightarrow g$, between simplicial space maps $f, g: S \rightarrow S^{\prime}$, determines a homotopy $\mathrm{B} H: \mathrm{B} f \Rightarrow \mathrm{B} g$ [32, Corollary 11.10]. For instance, by regarding a set as a discrete space, the (Milnor's) geometric realization of a simplicial set $S: \Delta^{\mathrm{op}} \rightarrow$ Set is B $S$. If $f, g: S \rightarrow S^{\prime}$ are simplicial maps, between simplicial sets, then a simplicial homotopy $H: f \Rightarrow g$ amounts to the same thing [31, Proposition 6.2] as a simplicial map $H: S \times \Delta[1] \rightarrow S^{\prime}$ making this diagram commutative:

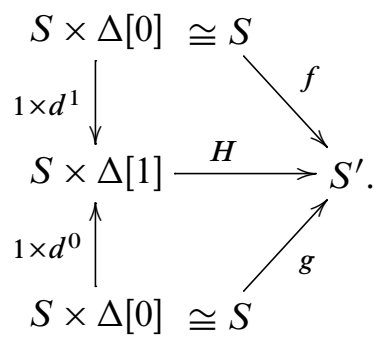

A weak homotopy equivalence of simplicial sets is a simplicial map whose geometric realization is a homotopy equivalence or, equivalently, induces isomorphisms in homotopy groups. 
A bisimplicial set is a functor $S: \Delta^{\mathrm{op}} \times \Delta^{\mathrm{op}} \rightarrow$ Set. This amounts to a family of sets $\left\{S_{p, q} ; p, q \geq 0\right\}$ together with horizontal and vertical face and degeneracy operators

$$
S_{p+1, q} \stackrel{s_{i}^{h}}{\longleftarrow} S_{p, q} \stackrel{d_{i}^{h}}{\longrightarrow} S_{p-1, q}, \quad S_{p, q+1} \stackrel{s_{j}^{v}}{\longleftarrow} S_{p, q} \stackrel{d_{j}^{v}}{\longrightarrow} S_{p, q-1},
$$

with $0 \leq i \leq p$ and $0 \leq j \leq q$ respectively, such that, for all $p$ and $q$, both $S_{p, *}$ and $S_{*, q}$ are simplicial sets and the horizontal operators commute with the vertical ones. Note that, on the one hand, any bisimplicial set $S$ provides two simplicial objects in the category of simplicial sets: the horizontal one $S^{h}:[p] \mapsto S_{p, *}$ and the vertical one $S^{v}:[q] \mapsto S_{*, q}$. Then, by taking realization, $S$ gives rise to two simplicial spaces $\mathrm{B} S^{h}:[p] \mapsto \mathrm{B} S_{p, *}$ and $\mathrm{B} S^{v}:[q] \mapsto \mathrm{B} S_{*, q}$, respectively. On the other hand, by composing with the diagonal functor diag: $\Delta^{\mathrm{op}} \rightarrow \Delta^{\mathrm{op}} \times \Delta^{\mathrm{op}}$, the bisimplicial set $S$ also provides another simplicial set $\operatorname{diag} S:[n] \mapsto S_{n, n}$, whose face and degeneracy operators are given in terms of those of $S$ by the formulas $d_{i}=d_{i}^{h} d_{i}^{v}$ and $s_{i}=s_{i}^{h} s_{i}^{v}$, respectively. It is known (eg [35, Lemma in page 86]) that there are natural homeomorphisms

$$
\mathrm{BB} S^{h} \cong \operatorname{Bdiag} S \cong \mathrm{BB} S^{v} .
$$

It is a relevant fact that, if $f: S \rightarrow S^{\prime}$ is a bisimplicial map such that the induced maps $f_{p, *}: S_{p, *} \rightarrow S_{p, *}^{\prime}\left(\right.$ resp. $f_{*, q}: S_{*, q} \rightarrow S_{*, q}^{\prime}$ ) are weak homotopy equivalences for all $p$ (resp. $q$ ), then so is the map $\operatorname{diag} f: \operatorname{diag} S \rightarrow \operatorname{diag} S^{\prime}$ (see Bousfield and Kan [4, Chapter XII, 4.2 and 4.3] or Goerss and Jardine [19, IV, Proposition 1.7], for example).

We shall also use the bar construction on a bisimplicial set $\bar{W} S$, also called its codiagonal or total complex. Let us recall that the functor

\section{$\bar{W}:$ Bisimpl.Set $\rightarrow$ Simpl.Set}

is the right Kan extension along the ordinal sum functor $\Delta \times \Delta \rightarrow \Delta,([p],[q]) \mapsto$ $[p+1+q]$. For any given bisimplicial set $S, \bar{W} S$ can be described as follows $[1$, Section III]: the set of $p$-simplices of $\bar{W} S$ is

$$
\left\{\left(t_{0, p} \ldots, t_{p, 0}\right) \in \prod_{m=0}^{p} S_{m, p-m} \mid d_{0}^{v} t_{m, p-m}=d_{m+1}^{h} t_{m+1, p-m-1}, 0 \leq m<p\right\}
$$

and, for $0 \leq i \leq p$, the faces and degeneracies of a $p$-simplex are given by

$$
\begin{aligned}
d_{i}\left(t_{0, p} \ldots, t_{p, 0}\right) & =\left(d_{i}^{v} t_{0, p}, \ldots, d_{i}^{v} t_{i-1, p-i+1}, d_{i}^{h} t_{i+1, p-i-1}, \ldots, d_{i}^{h} t_{p, 0}\right), \\
s_{i}\left(t_{0, p} \ldots, t_{p, 0}\right) & =\left(s_{i}^{v} t_{0, p}, \ldots, s_{0}^{v} t_{i, p-i}, s_{i}^{h} t_{i, p-i}, \ldots, s_{i}^{h} t_{p, 0}\right) .
\end{aligned}
$$

For any bisimplicial set $S$, there is a natural weak homotopy equivalence [13; 14]

$$
\Phi: \operatorname{diag} S \rightarrow \bar{W} S,
$$


which carries a $p$-simplex $t_{p, p} \in \operatorname{diag} S$ to

$$
\Phi t_{p, p}=\left(\left(d_{1}^{h}\right)^{p} t_{p, p},\left(d_{2}^{h}\right)^{p-1} d_{0}^{v} t_{p, p}, \ldots,\left(d_{m+1}^{h}\right)^{p-m}\left(d_{0}^{v}\right)^{m} t_{p, p}, \ldots,\left(d_{0}^{v}\right)^{p} t_{p, p}\right) .
$$

\subsection{The classifying space of a small category}

In Quillen's development of $K$-theory, the higher $K$-groups are defined as the homotopy groups of a topological classifying space, $\mathrm{BC}$, functorially associated to a small category $\mathcal{C}$, the geometric realization of its so-called nerve (see Grothendieck [21]). This nerve is a simplicial set encoding the structure of the category in terms of its faces and degeneracies, and it can be easily described by means of two isomorphic constructions:

On one hand, the nerve of a category $\mathcal{C}$ can be defined as the simplicial set

$$
\mathrm{NC}: \Delta^{\mathrm{op}} \longrightarrow \text { Set, }
$$

whose set of $p$-simplices

$$
\begin{aligned}
\mathrm{NC}_{p} & =\bigsqcup_{\left(x_{0}, \ldots, x_{p}\right) \in \mathrm{Ob} \mathcal{C}^{p+1}} \mathcal{C}\left(x_{1}, x_{0}\right) \times \mathcal{C}\left(x_{2}, x_{1}\right) \times \cdots \times \mathcal{C}\left(x_{p}, x_{p-1}\right), \\
\mathrm{NC}_{0} & =\mathrm{Ob} \mathcal{C},
\end{aligned}
$$

consists of length $p$ sequences of composable morphisms in $\mathcal{C}$

$$
x_{0} \longleftarrow u_{1} x_{1} \longleftarrow \text { u } \cdots \stackrel{u_{p}}{\longleftarrow} x_{p} .
$$

The face and degeneracy operators are defined by the well-known formulas:

$$
\begin{aligned}
& d_{i}\left(u_{1}, \ldots, u_{p}\right)= \begin{cases}\left(u_{2}, \ldots, u_{p}\right) & \text { if } i=0, \\
\left(u_{1}, \ldots, u_{i} u_{i+1}, \ldots, u_{p}\right) & \text { if } 0<i<p, \\
\left(u_{1}, \ldots, u_{p-1}\right) & \text { if } i=p,\end{cases} \\
& s_{i}\left(u_{1}, \ldots, u_{p}\right)=\left(u_{1}, \ldots, u_{i}, 1_{x_{i}}, u_{i+1}, \ldots, u_{p}\right) .
\end{aligned}
$$

On the other hand, let

$$
\begin{aligned}
\Delta \mathcal{C}: & \Delta^{\mathrm{op}} \longrightarrow \text { Set }, \\
& {[p] \mapsto \operatorname{Func}([p], \mathcal{C}), }
\end{aligned}
$$

be the simplicial set whose $p$ simplices are the functors $F:[p] \rightarrow \mathcal{C}$ or, equivalently, tuples of arrows in $\mathcal{C}$

$$
F=\left(F j \stackrel{F_{i, j}}{\longrightarrow} F i\right)_{0 \leq i \leq j \leq p}
$$

such that $F_{i, j} F_{j, k}=F_{i k}$, for $i \leq j \leq k$ and $F_{i, i}=1_{F i}$. 
As there is quite an obvious simplicial isomorphism

$$
\begin{aligned}
& \Delta \mathcal{C} \cong \mathrm{NC}, \\
& F \mapsto\left(F 0 \stackrel{F_{0,1}}{\longleftarrow} F 1 \stackrel{F_{1,2}}{\longleftarrow} \cdots \stackrel{F_{p-1, p}}{\longleftarrow} F p\right)
\end{aligned}
$$

both simplicial sets $\Delta \mathcal{C}$ and $\mathrm{NC}$ are usually identified, and hereafter we will also do so. However, note that $\Delta \mathcal{C}$ has a more pleasing geometric interpretation than $\mathrm{NC}$, since a $p$-simplex of $\Delta \mathcal{C}$ can be thought of as the 1-skeleton of an oriented standard $p$-simplex with objects $F i$ of $\mathcal{C}$ placed on the vertices and arrows $F_{i, j}: F j \rightarrow F i$ placed on the edges for $0 \leq i<j \leq p$, and the requirement that every triangle

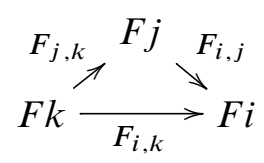

be commutative.

For instance, note that $\mathrm{N}[n] \cong \Delta[n]$ is the simplicial standard $n$-simplex, and $\mathrm{B}[n]=\Delta_{n}$ is the standard $n$-dimensional affine simplex. When a monoid (or group) $\mathcal{M}$ is regarded as a category with only one object, then $\mathrm{B} \mathcal{M}$ is its classifying space in the traditional sense. Therefore, many weak homotopy types thus occur, since every pathconnected space has the weak homotopy type of the classifying space of a monoid [16, Theorem 3.5]. Moreover, any CW-complex is homotopy equivalent to the classifying space of a small category, as Quillen showed [35]: The category of simplices $\int_{\Delta} S$, of a simplicial set $S$, has as objects pairs $(p, x)$ where $p \geq 0$ and $x$ is a $p$-simplex of $S$; and arrow $\alpha:(p, x) \rightarrow(q, y)$ is an arrow $\alpha:[p] \rightarrow[q]$ in $\Delta$ with the property $x=\alpha^{*} y$. Then there exists a homotopy equivalence $|S| \simeq \mathrm{B} \int_{\Delta} S$ between the geometric realization of $S$ and the classifying space of $\int_{\Delta} S$ (this result is, in fact, a very particular case of the homotopy colimit theorem of Thomason [43]). If $X$ is any CW-complex and we take $S=S X$, the total singular complex of $X$, then $X \simeq|S X| \simeq B \int_{\Delta} S X$.

The nerve construction on categories gives a fully faithful functor, embedding the category Cat into the category of simplicial sets. Moreover, this functor obviously commutes with products and, as pointed out by Segal [36, Proposition 2.1], these facts imply that, for any two functors $F, G: \mathcal{C} \rightarrow \mathcal{D}$, the functor nerve defines a bijection

Nat. transformations $(F, G) \cong \operatorname{Simpl}$ homotopies $(\Delta F, \Delta G)$,

between the set of natural transformations $\alpha: F \Rightarrow G$ and the set of simplicial homotopies between the induced simplicial maps on the nerves: Interpret $\alpha$ as a functor $\alpha: \mathcal{C} \times[1] \rightarrow \mathcal{D}$. Hence, what $\alpha$ induces is a simplicial map $\Delta \alpha: \Delta \mathcal{C} \times \Delta[1] \rightarrow \Delta \mathcal{D}$, 
that is, a simplicial homotopy. As a consequence, functors related by some natural transformation go to homotopic cellular maps on classifying spaces. In particular, if a functor $F: \mathcal{C} \rightarrow \mathcal{D}$ has a left or right adjoint, the induced map $\mathrm{B} F: \mathrm{BC} \rightarrow \mathrm{B} \mathcal{D}$ is a homotopy equivalence.

\subsection{The classifying space of a diagram of categories}

In [36], Segal extended Milnor's geometric realization process to simplicial (compactly generated topological) spaces and provided, for instance, the notion of classifying spaces for simplicial categories $\mathcal{C}: \Delta^{\mathrm{op}} \rightarrow$ Cat: By replacing each category $\mathcal{C}_{p}, p \geq 0$, by its classifying space $\mathrm{BC} \mathcal{C}_{p}$, one obtains a simplicial space, whose Segal realization is, by definition, the classifying space $\mathrm{BC}$ of the simplicial category $\mathcal{C}$, that is,

$$
\mathrm{BC}=\mathrm{B}\left([p] \mapsto \mathrm{BC}_{p}\right) .
$$

But note, as an instance of the homeomorphisms (1), that there is a natural homeomorphism

$$
\mathrm{BC} \cong \mathrm{Bdiag} \Delta \mathcal{C}
$$

with the geometric realization of the simplicial set diagonal of the bisimplicial set obtained by composing $\mathcal{C}: \Delta^{\text {op }} \rightarrow$ Cat with the nerve functor $\Delta$ : Cat $\rightarrow$ Simpl.Set, that is,

$$
\begin{aligned}
\Delta \mathcal{C}: & \Delta^{\mathrm{op}} \times \Delta^{\mathrm{op}} \longrightarrow \text { Set } \\
& ([p],[q]) \longmapsto \operatorname{Func}\left([q], \mathcal{C}_{p}\right) .
\end{aligned}
$$

Segal's construction above, for simplicial categories, is actually a particular case of the more general notion of classifying space for arbitrary diagrams of categories: If $\mathcal{C}: I^{\mathrm{op}} \rightarrow$ Cat is a functor, where $I$ is any category, then its classifying space

$$
\mathrm{BC}=\mathrm{B}_{\operatorname{hocolim}_{I}} \Delta \mathcal{C},
$$

is given through the homotopy colimit construction by Bousfield and Kan [4, Section XII], of the diagram of simplicial sets $\Delta \mathcal{C}: I^{\text {op }} \rightarrow$ Simpl.Set, obtained by composing $\mathcal{C}$ with the nerve functor. That is, the simplicial set

$$
\begin{aligned}
\operatorname{hocolim}_{I} \Delta \mathcal{C}: & \Delta^{\mathrm{op}} \longrightarrow \text { Set. } \\
& {[p] \mapsto \bigsqcup_{[p] \rightarrow I} \operatorname{Func}\left([p], \mathcal{C}_{\sigma 0}\right) }
\end{aligned}
$$

Since, for any simplicial category $\mathcal{C}: \Delta^{\mathrm{op}} \rightarrow$ Cat, there is a natural weak homotopy equivalence of simplicial sets [4, XII, 4.3],

$$
\operatorname{hocolim}_{\Delta} \Delta \mathcal{C} \stackrel{\sim}{\longrightarrow} \operatorname{diag} \Delta \mathcal{C},
$$


then both constructions (8) and (10) for the classifying space $\mathrm{BC}$ of a simplicial category $\mathcal{C}$ coincide up to a natural homotopy equivalence.

\subsection{Some bicategorical conventions}

We employ the standard nomenclature concerning bicategories and refer to Bénabou [3], Duskin [15], Gordon, Power and Street [20], Gurski [22] or Street [41] for the background. For the sake of clarity, we state the following:

A small bicategory, $\mathcal{C}$, provides us with the following data: (i) a set $\mathrm{Ob} \mathcal{C}$ of objects (or $0-$ cells) of $\mathcal{C}$; (ii) for each ordered pair of objects $(y, x)$ of $\mathcal{C}$, a category $\mathcal{C}(y, x)$ whose objects $u: y \rightarrow x$ are called morphisms (or 1-cells) of $\mathcal{C}$ with source $y$ and target $x$, and whose arrows $\alpha: u \Rightarrow u^{\prime}$ are called deformations (or 2-cells) of $\mathcal{C}$ and are usually depicted as

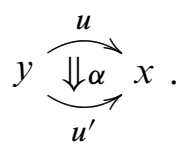

The composition of deformations in each category $\mathcal{C}(y, x)$ is called the vertical composition and will be denoted by $\beta \cdot \alpha$; (iii) for every triplet $(z, y, x)$ of objects of $\mathcal{C}$, a functor $\mathcal{C}(y, x) \times \mathcal{C}(z, y) \stackrel{\circ}{\rightarrow} \mathcal{C}(z, x)$,

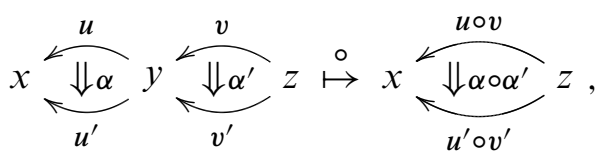

called horizontal composition; (iv) for every object $x$ of $\mathcal{C}$, a distinguished morphism $1_{x}: x \rightarrow x$, called the identity of $x ;(\mathrm{v})$ invertible deformations

$$
\boldsymbol{a}_{u, v, w}: u \circ(v \circ w) \stackrel{\sim}{\Rightarrow}(u \circ v) \circ w,
$$

called the associativity constraints, which are natural in $(u, v, w) \in \mathcal{C}(y, x) \times \mathcal{C}(z, y) \times$ $\mathcal{C}(t, z)$; and (vi) invertible deformations

$$
\boldsymbol{l}_{u}: 1_{x} \circ u \stackrel{\sim}{\Rightarrow} u \quad \text { and } \quad \boldsymbol{r}_{u}: u \circ 1_{y} \stackrel{\sim}{\Rightarrow} u,
$$

called identity constraints, which are natural in $u \in \mathcal{C}(y, x)$. These constraints are required to be coherent, in the sense that the following pentagons are commutative

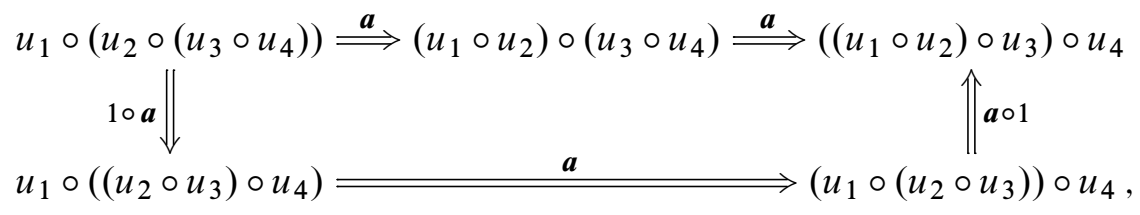


and the following triangles are commutative:

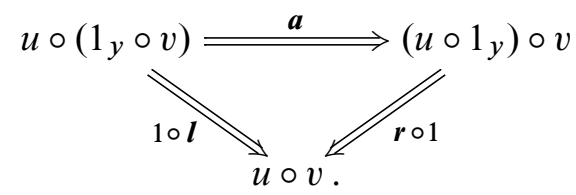

In any bicategory, for any object $x$, the equality

$$
l_{1_{x}}=\boldsymbol{r}_{1_{x}}
$$

holds, and, for any two morphisms $v: z \rightarrow y$ and $u: y \rightarrow x$, the two triangles below commute.
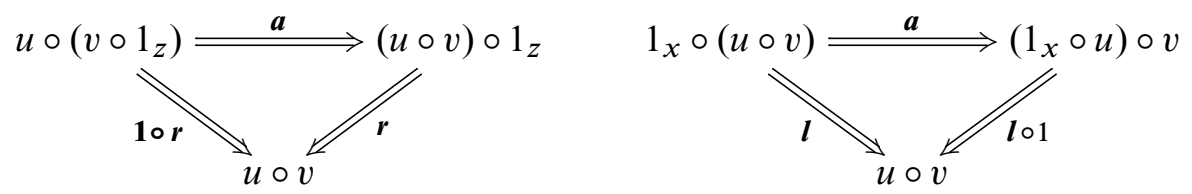

A bicategory in which all the constraints are identities is a 2-category, that is, just a category enriched in the category Cat of small categories. As each category $\mathcal{C}$ can be considered as a 2-category in which all deformations are identities, that is, in which each category $\mathcal{C}(x, y)$ is discrete, several times throughout the paper categories are considered as special bicategories.

If $\mathcal{B}, \mathcal{C}$ are bicategories, then a lax functor $F=(F, \widehat{F}): \mathcal{B} \rightsquigarrow \mathcal{C}$ consists of: (i) a mapping $F: \mathrm{Ob} \mathcal{B} \rightarrow \mathrm{Ob} \mathcal{C}$; (ii) for each ordered pair of objects $(y, x)$ of $\mathcal{B}$, a functor $F: \mathcal{B}(y, x) \rightarrow \mathcal{C}(F y, F x)$; (iii) deformations $\hat{F}_{u, v}: F u \circ F v \Rightarrow F(u \circ v)$ that are natural in $(u, v) \in \mathcal{B}(y, x) \times \mathcal{B}(z, y)$; and (iv) for each object $x$ of $\mathcal{B}$, a deformation $\widehat{F}_{x}: 1_{F x} \Rightarrow F 1_{x}$. All these data are subject to the coherence commutativity conditions:

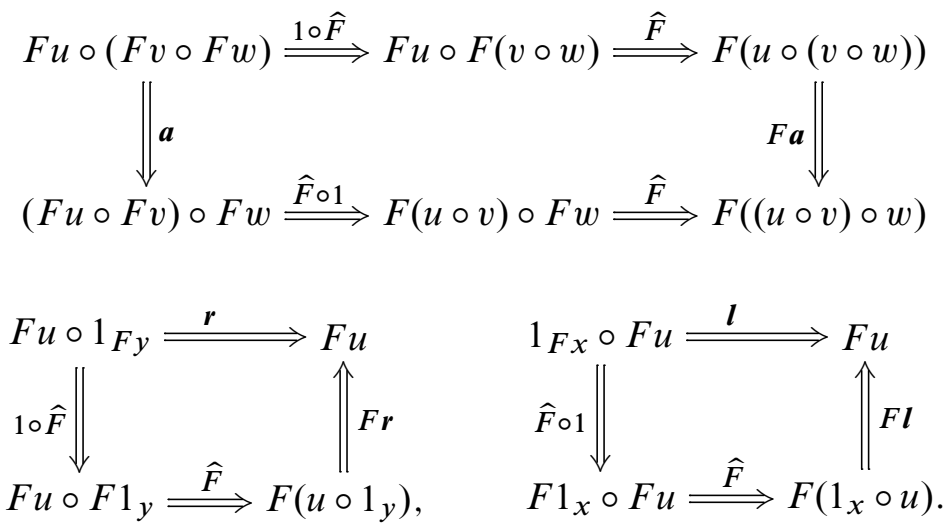


Replacing the structure deformations above with $\widehat{F}_{u, v}: F(u \circ v) \Rightarrow F u \circ F v$ and $\widehat{F}_{x}: F\left(1_{x}\right) \Rightarrow 1_{F x}$, we have the notion of oplax functor $F: \mathcal{B} \rightsquigarrow \mathcal{C}$.

A lax functor is termed a pseudo functor or a homomorphism whenever all the structure constraints $\widehat{F}_{u, v}: F u \circ F v \Rightarrow F(u \circ v)$ and $\widehat{F}_{x}: 1_{F x} \Rightarrow F\left(1_{x}\right)$ are invertible. When these deformations are all identities, then $F$ is called a 2 -functor and is written as $F: \mathcal{B} \rightarrow \mathcal{C}$. If the unit constraints $\widehat{F}_{x}$ are all identities, then the lax functor is qualified as (strictly) unitary or normal.

The composition of lax functors $F: \mathcal{A} \rightsquigarrow \mathcal{B}$ and $G: \mathcal{B} \rightsquigarrow \mathcal{C}$ will be denoted by juxtaposition, that is, $G F: \mathcal{A} \rightsquigarrow \mathcal{C}$. Recall that its constraints are obtained from those of $F$ and $G$ by the rule $\widehat{G F}=G \widehat{F} \cdot \widehat{G} F$; that is, by the compositions

$$
\begin{aligned}
& \widehat{G F}_{u, v}: G F u \circ G F v \stackrel{\widehat{G}_{F u, F v}}{\Longrightarrow} G(F u \circ F v) \stackrel{G \widehat{F}_{u, v}}{\Longrightarrow} G F(u \circ v), \\
& \widehat{G F}_{x}: 1_{G F x} \stackrel{\widehat{G}_{F x}}{\Longrightarrow} G 1_{F x} \stackrel{G \widehat{F}_{x}}{\longrightarrow} G F 1_{x} .
\end{aligned}
$$

The composition of lax functors is associative and unitary, so that the category of bicategories and lax functors is defined. The subcategory of bicategories with homomorphisms between them will be denoted by Bicat.

If $F, F^{\prime}: \mathcal{B} \rightsquigarrow \mathcal{C}$ are lax functors, then we follow the convention of [20] in what is meant by a lax transformation $\alpha=(\alpha, \hat{\alpha}): F \Rightarrow F^{\prime}$. Thus, $\alpha$ consists of morphisms $\alpha x: F x \rightarrow F^{\prime} x, x \in \mathrm{Ob} \mathcal{B}$, and deformations

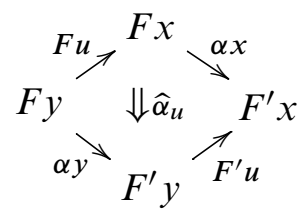

that are natural on morphisms $u: y \rightarrow x$, subject to the usual two commutativity axioms:

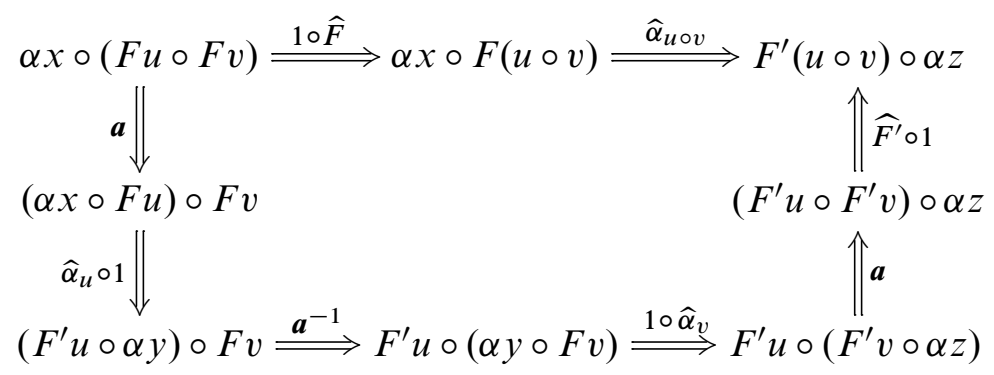




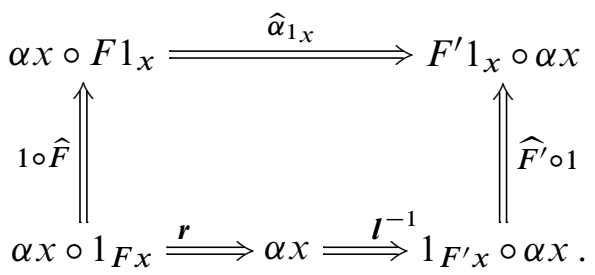

Replacing the structure deformation above with $\hat{\alpha}_{u}: F^{\prime} u \circ \alpha y \Rightarrow \alpha x \circ F u$, we have the notion of oplax transformation $\alpha: F \Rightarrow F^{\prime}$.

\subsection{The classifying space of a lax diagram of categories}

In nature, actual functors $\mathcal{C}: I^{\mathrm{op}} \rightarrow$ Cat are rare, but pseudofunctors are ubiquitous. The above construction (10), of classifying spaces for diagrams of categories, was extended to lax diagrams of categories by Thomason [43] using methods by Grothendieck. Recall that, for any given small category $I$, a lax diagram of categories means a lax functor

$$
\mathcal{C}=(\mathcal{C}, \widehat{\mathcal{C}}): I^{\text {op }} \rightsquigarrow \mathbf{C a t},
$$

to the 2-category Cat of small categories, functors, and natural transformations. So $\mathcal{C}$ is a system of data consisting of a category $\mathcal{C}_{i}$ for each object $i$ of $I$, a functor $a^{*}: \mathcal{C}_{i} \rightarrow \mathcal{C}_{j}$ for each arrow $a: j \rightarrow i$ of $I$, a natural transformation $\widehat{\mathcal{C}}=\widehat{\mathcal{C}}_{a, b}: b^{*} a^{*} \Rightarrow(a b)^{*}$, for each two composable arrows $b: k \rightarrow j$ and $a: j \rightarrow i$ in $I$, and a natural transformation $\widehat{\mathcal{C}}=\widehat{\mathcal{C}}_{i}: 1_{\mathcal{C}_{i}} \Rightarrow 1_{i}^{*}$, for each object $i$ of $I$. These must satisfy the conditions that, for $c: \ell \rightarrow k, b: k \rightarrow j$ and $a: j \rightarrow i$, the following diagram commutes:

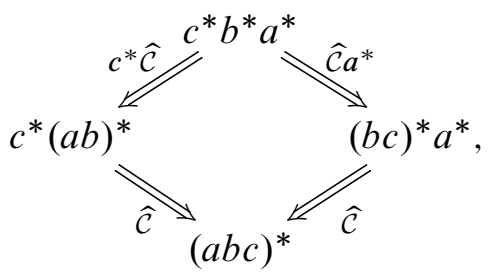

and for $a: j \rightarrow i$ the compositions

$$
a^{*} \stackrel{\hat{\mathcal{C}} a^{*}}{\Longrightarrow} 1_{j}^{*} a^{*} \stackrel{\widehat{\mathcal{c}}}{\Longrightarrow} a^{*} \quad \text { and } \quad a^{*} \stackrel{a^{*} \widehat{c}}{\Longrightarrow} a^{*} 1_{i}^{*} \stackrel{\widehat{\mathcal{c}}}{\Longrightarrow} a^{*}
$$

are both the identity transformation on the functor $a^{*}: \mathcal{C}_{i} \rightarrow \mathcal{C}_{j}$.

The so-called Grothendieck construction on a lax diagram of categories $\mathcal{C}: I^{\mathrm{op}} \rightsquigarrow$ Cat, denoted by

$$
\int_{I} \mathcal{C}
$$


is the category whose objects are pairs $(x, i)$, where $i$ is an object of $I$ and $x$ is one of $\mathcal{C}_{i}$; a morphism $(u, a):(y, j) \rightarrow(x, i)$ in $\int_{I} \mathcal{C}$ is a pair of morphisms where $a: j \rightarrow i$ in $I$ and $u: y \rightarrow a^{*} x$ in $\mathcal{C}_{j}$. If $(v, b):(z, k) \rightarrow(y, j)$ is another morphism in $\int_{I} \mathcal{C}$, then we have the morphisms

$$
z \stackrel{v}{\longrightarrow} b^{*} y \stackrel{b^{*} u}{\longrightarrow} b^{*} a^{*} x \stackrel{\widehat{\mathcal{C}} x}{\longrightarrow}(a b)^{*} x
$$

in $\mathcal{C}_{k}$, and the composition of $(v, b)$ with $(u, a)$ is defined by

$$
(u, a)(v, b)=\left(\hat{\mathcal{C}} x \cdot b^{*} u \cdot v, a b\right):(z, k) \rightarrow(x, i) .
$$

The identity morphism of an object $(x, i)$ is $\left(\hat{\mathcal{C}}_{i} x: x \longrightarrow 1_{i}^{*} x, 1_{i}\right)$.

Then, the classifying space of the lax diagram $\mathcal{C}: I^{\text {op }} \rightsquigarrow$ Cat, BC , is defined to be the classifying space of its Grothendieck construction, that is,

$$
\mathrm{BC}=\mathrm{B} \int_{I} \mathcal{C}
$$

Thomason's homotopy colimit theorem [43, Theorem 1.2] states the following:

Theorem 2.1 Let $\mathcal{C}: I^{\mathrm{op}} \rightarrow$ Cat be a functor. There is a natural weak homotopy equivalence

$$
\eta: \operatorname{hocolim}_{I} \mathrm{NC} \longrightarrow \mathrm{N} \int_{I} \mathcal{C}
$$

Therefore, both definitions (10) and (18), for the classifying space $\mathrm{BC}$ of a diagram of categories $\mathcal{C}: I^{\mathrm{op}} \rightarrow$ Cat, lead to the same space, up to a natural homotopy equivalence. Furthermore, the construction of $\mathrm{BC}$ is consistent with the so-called Street rectification process, $\mathcal{C} \mapsto \mathcal{C}^{\prime}[39]$ (see also May [33, Theorem 3.4]), which associates to any lax diagram of categories $\mathcal{C}: I^{\text {op }} \rightsquigarrow$ Cat a homotopy equivalent strict diagram $\mathcal{C}^{\prime}: I^{\text {op }} \rightarrow$ Cat, that is, such that there is a natural homotopy equivalence [43, Lemma 3.2.5]

$$
\mathrm{BC} \simeq \mathrm{BC}^{\prime}
$$

The Grothendieck construction on lax diagrams $\mathcal{C}: I^{\text {op }} \rightsquigarrow$ Cat is natural both in $I$ and $\mathcal{C}$. Recall that, given lax diagrams $\mathcal{C}, \mathcal{D}: I^{\mathrm{op}} \rightsquigarrow$ Cat, a lax morphism (or oplax transformation)

$$
F=(F, \widehat{F}): \mathcal{C} \rightsquigarrow \mathcal{D},
$$


is a system of data consisting of a functor $F_{i}: \mathcal{C}_{i} \rightarrow \mathcal{D}_{i}$, for each object $i$ of $I$, and a natural transformation $\widehat{F}_{a}: F_{j} a^{*} \Rightarrow a^{*} F_{i}$,

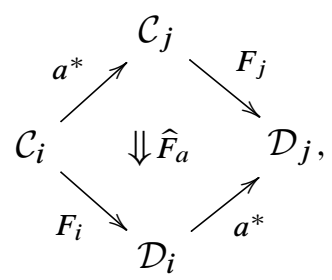

for each morphism $a: j \rightarrow i$ in $I$. These are subject to the conditions that the following diagrams commute
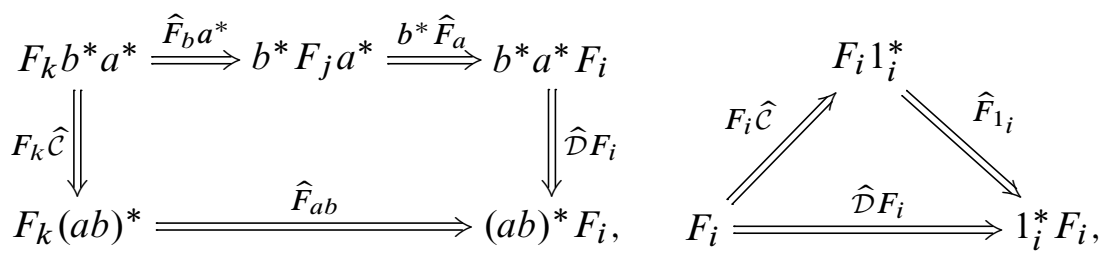

for every pair of composable morphisms $b: k \rightarrow j$ and $a: j \rightarrow i$ and any object $i$ in $I$. Any lax morphism $F: \mathcal{C} \rightsquigarrow \mathcal{D}$ induces a functor $\int_{I} F: \int_{I} \mathcal{C} \rightarrow \int_{I} \mathcal{D}$, whence a cellular map

$$
\mathrm{B} F: \mathrm{BC} \rightarrow \mathrm{B} \mathcal{D},
$$

defined on objects by $F_{*}(x, i)=\left(F_{i} x, i\right)$. For a morphism $(u, a):(y, j) \rightarrow(x, i)$ in $\int_{I} \mathcal{C}$, we have the composable morphisms in $\mathcal{D}_{j}$

$$
F_{j} y \stackrel{F_{j} u}{\longrightarrow} F_{j} a^{*} x \stackrel{\widehat{F}_{a} x}{\longrightarrow} a^{*} F_{i} x,
$$

and $F_{*}(u, a)=\left(\widehat{F}_{a} x \cdot F_{j} u, a\right)$. A main result by Thomason [43, Corollary 3.3.1] states the following:

Theorem 2.2 If $F: \mathcal{C} \rightsquigarrow \mathcal{D}$ is a lax morphism between lax diagrams $\mathcal{C}, \mathcal{D}: I^{\mathrm{op}} \rightsquigarrow$ Cat such that the induced maps $B F_{i}: \mathrm{BC}_{i} \rightarrow \mathrm{BD}_{i}$ are homotopy equivalences, for all objects $i$ of $I$, then the induced map $\mathrm{B} F: \mathrm{BC} \rightarrow \mathrm{BD}$ is a homotopy equivalence.

\section{The pseudosimplicial nerve and the classifying space of a bicategory}

Let $\mathcal{C}$ be any given bicategory. When $\mathcal{C}$ is strict (ie, a 2 -category), then the nerve construction (3) actually works by giving a simplicial category $\underline{\mathrm{NC}}: \Delta^{\text {op }} \rightarrow$ Cat, whose 
Segal's classifying space (8), or (9), is usually taken to be the classifying space of the 2-category (see Bullejos and Cegarra [6], Hinich and Schechtman [24], Moerdijk and Svensson [34], Tillmann [45; 44] or Thomason [43], for examples). For an arbitrary $\mathcal{C}$, its classifying space is defined in a similar way as for the strict case; however, the process is more complicated since the horizontal composition in a bicategory is in general not associative and not unitary (which is crucial for constructing the simplicial category $\underline{\mathrm{NC}}$ ) but it is only so up to coherent isomorphisms. This "defect" has the effect of forcing one to deal with the classifying space of a nerve of $\mathcal{C}$, which is not simplicial but only up to coherent isomorphisms: the (normal) pseudosimplicial category

$$
\underline{\mathrm{NC}}: \Delta^{\mathrm{op}} \rightsquigarrow \mathbf{C a t}
$$

whose category of $p$-simplices is

$$
\underline{\mathrm{N}}_{p}=\bigsqcup_{\left(x_{0}, \ldots, x_{p}\right) \in \mathrm{Ob} \mathcal{C}^{p+1}} \mathcal{C}\left(x_{1}, x_{0}\right) \times \mathcal{C}\left(x_{2}, x_{1}\right) \times \cdots \times \mathcal{C}\left(x_{p}, x_{p-1}\right),
$$

where a typical arrow is a string of deformations in $\mathcal{C}$

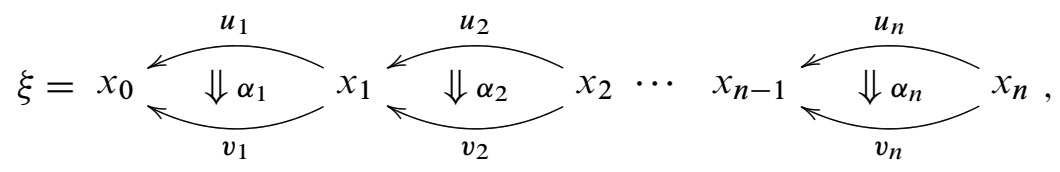

and $\underline{\mathrm{NC}}_{0}=\mathrm{Ob} \mathcal{C}$, as a discrete category.

The face and degeneracy functors are defined in the standard way by the formulas (5), both for morphisms and deformations, substituting juxtaposition with the symbol $\circ$, used for the horizontal composition in $\mathcal{C}$. That is, $d_{0}(\xi)=\left(\alpha_{2}, \ldots, \alpha_{n}\right), d_{1}(\xi)=$ $\left(\alpha_{1} \circ \alpha_{2}, \ldots, \alpha_{n}\right)$, and so on.

If $a:[q] \rightarrow[p]$ is any nonidentity map in $\Delta$, then we write $a$ in the (unique) form (see May [31], for example) $a=d^{i_{1}} \cdots d^{i_{s}} s^{j_{1}} \cdots s^{j_{t}}$, where $0 \leq i_{s}<\cdots<i_{1} \leq p$, $0 \leq j_{1}<\cdots<j_{t} \leq q$ and $q+s=p+t$, and the induced functor $a^{*}: \underline{\mathrm{N}}_{p} \mathcal{C} \rightarrow \underline{\mathrm{N}}_{q} \mathcal{C}$ is defined by $a^{*}=s_{j_{t}} \cdots s_{j_{1}} d_{i_{s}} \cdots d_{i_{1}}$. Note that $d_{j} d_{i}=d_{i} d_{j+1}$ for $i \leq j$, unless $i=j$ and $1 \leq i \leq p-2$, in which case the associativity constraint of $\mathcal{C}$ gives a canonical natural isomorphism

$$
d_{i} d_{i} \cong d_{i} d_{i+1} \text {. }
$$

Similarly, all the equalities $d_{0} s_{0}=1, d_{p+1} s_{p}=1, d_{i} s_{j}=s_{j-1} d_{i}$ if $i<j$ and $d_{i} s_{j}=$ $s_{j} d_{i-1}$ if $i>j+1$, hold, and the unit constraints of $\mathcal{C}$ give canonical isomorphisms

$$
d_{i} s_{i} \cong 1, \quad d_{i} s_{i+1} \cong 1
$$


Then it is a fact that this family of natural isomorphisms (23) and (24) uniquely determines a whole system of natural isomorphisms

$$
b^{*} a^{*} \cong(a b)^{*},
$$

one for each pair of composable maps in $\Delta, b:[n] \rightarrow[q]$ and $a:[q] \rightarrow[p]$, such that the assignments $a \mapsto a^{*}, 1_{[p]} \mapsto 1_{\underline{N} \mathcal{C}_{p}}$, together with these isomorphisms $b^{*} a^{*} \cong(a b)^{*}$, give the data for the pseudosimplicial category (21), $\underline{N C}: \Delta^{\text {op }} \rightsquigarrow$ Cat. This fact can be easily proven by using Jardine's supercoherence theorem [25, Corollary 1.6], since the commutativity of the seventeen diagrams of supercoherence, (1.4.1)-(1.4.17) in [25], easily follows from the coherence conditions (13), (14), (15) and (16).

Recalling the construction (18) for the classifying spaces of lax diagrams of categories, we state the following:

Definition 3.1 The classifying space $\mathrm{BC}$, of a bicategory $\mathcal{C}$, is the classifying space of its pseudosimplicial nerve (21), $\underline{N} \mathcal{C}: \Delta^{\text {op }} \rightsquigarrow$ Cat, that is,

$$
\mathrm{BC}=\mathrm{B} \int_{\Delta} \underline{\mathrm{NC}} .
$$

Remark 3.2 Let $\mathcal{C}$ be a 2 -category. Then, its pseudosimplicial nerve (21) is actually a simplicial category

$$
\underline{\mathrm{NC}}: \Delta^{\mathrm{op}} \rightarrow \text { Cat, }
$$

and there are natural homotopy equivalences

$$
\mathrm{BC} \stackrel{(19)}{\simeq}\left|\operatorname{hocolim}_{\Delta} \mathrm{NN} \underline{C}\right| \stackrel{(12)}{\simeq}|\operatorname{diagN} \underline{\mathrm{NC}}| \stackrel{(9)}{\simeq} \mathrm{BNC},
$$

where $\underline{\mathrm{N} C}:([p],[q]) \mapsto \mathrm{N}\left(\underline{\mathrm{NC}}_{p}\right)_{q}$ is the bisimplicial set double nerve of the 2-category obtained by composing $\mathrm{NC}$ with the functor nerve of categories, and $\mathrm{BNC}$ is the classifying space (8) of the simplicial category $\underline{N} \mathcal{C}$, that is, the Segal realization of the simplicial space $[p] \mapsto \mathrm{BNC}_{p}$.

Remark 3.3 A monoidal (tensor) category $\mathcal{M}=(\mathcal{M}, \otimes, \mathrm{I}, \boldsymbol{a}, \boldsymbol{l}, \boldsymbol{r})$ [30] can be viewed as a bicategory

$$
\Omega^{-1} \mathcal{M}
$$

with only one object, say $*$, the objects $u$ of $\mathcal{M}$ as morphisms $u: * \rightarrow *$ and the morphisms of $\mathcal{M}$ as deformations. Thus, $\Omega^{-1} \mathcal{M}(*, *)=\mathcal{M}$, and it is the horizontal composition of morphisms and deformations given by the tensor functor $\otimes: \mathcal{M} \times \mathcal{M} \rightarrow \mathcal{M}$. The identity at the object is $1_{*}=\mathrm{I}$, the unit object of the monoidal 
category, and the associativity, left unit, and right unit constraints for $\Omega^{-1} \mathcal{M}$ are just those of the monoidal category, that is, $\boldsymbol{a}, \boldsymbol{l}$ and $\boldsymbol{r}$, respectively.

The pseudosimplicial nerve of the bicategory $\Omega^{-1} \mathcal{M}$, as in (21), is exactly the pseudosimplicial category that the monoidal category defines by the reduced bar construction; that is, the pseudosimplicial category

$$
\underline{\mathrm{N}} \Omega^{-1} \mathcal{M}: \Delta^{\mathrm{op}} \rightsquigarrow \text { Cat, } \quad[p] \mapsto \mathcal{M}^{p},
$$

whose category of $p$-simplices is the $p$-fold power of the underlying category $\mathcal{M}$, with faces and degeneracy functors defined by analogy with those of the nerve of a monoid. Therefore, the classifying space of the monoidal category (see Jardine [25, Section 3], Hinich and Schechtman [24, Appendix] or Bullejos and Cegarra [7], for example) is just

$$
\mathrm{B} \Omega^{-1} \mathcal{M}
$$

the classifying space of the one object bicategory it defines.

The observation, due to Bénabou [3], that monoidal categories are essentially the same as bicategories with just one object, is known as the delooping principle, and the bicategory $\Omega^{-1} \mathcal{M}$ is called the delooping of the monoidal category [27, 2.10]. The reason for this terminology is the existence of a natural map

$$
\mathrm{B} \mathcal{M} \rightarrow \Omega\left(\mathrm{B} \Omega^{-1} \mathcal{M}, *\right)
$$

where $\mathrm{B} \mathcal{M}$ is the classifying space of the underlying category $\mathcal{M}$, which is, up to group completion, a homotopy equivalence (see Jardine [25, Propositions 3.5 and 3.8] or Bullejos and Cegarra [7, Corollary 4], for example; also, see Remark 6.6 for a proof therein). Then, the higher $K$-groups $K_{i}, i>0$, of the monoidal category $\mathcal{M}$ are the $(i+1)$-st homotopy groups of its classifying space $\mathrm{B} \Omega^{-1} \mathcal{M}$ (cf [37]). When the monoidal category has a given symmetry, then $\mathrm{B} \Omega^{-1} \mathcal{M}$ is precisely the space at level 1 of the $\Omega$-spectrum associated to the symmetric monoidal category (see Thomason [43, 4.2.2] or Jardine [25, 3.12], for example). The most striking instance is $\mathcal{M}=A$, the strict monoidal category with only one object defined by an abelian group $A$, where both compositions and tensor products are given by the addition in $A$; in this case, $\mathrm{B} A$ is a $K(A, 1)$-space, and $\Omega^{-1} A$ is a bicategory with only one object and only one arrow whose classifying space $\mathrm{B} \Omega^{-1} A$ is a $K(A, 2)$-space.

Any homomorphism $F: \mathcal{B} \rightsquigarrow \mathcal{C}$, between bicategories, gives rise to a morphism of supercoherent structures in the sense of Jardine [25], $F_{*}: \underline{\mathrm{N}} \mathcal{B} \rightarrow \underline{\mathrm{NC}}$, that, on a 
morphism $\xi$ as in (22), of the category of $p$-simplices of $\underline{N} \mathcal{B}$, acts by

$$
\xi \longmapsto F_{*} F x_{0} \underbrace{F u_{1}}_{\frac{F v_{1}}{\Downarrow F \alpha_{1}}} F x_{1} \underbrace{F u_{2}}_{\frac{F v_{2}}{\Downarrow F \alpha_{2}}} F x_{2} \cdots F x_{p-1} \frac{F u_{p}}{\underbrace{\Downarrow F \alpha_{p}}_{F v_{p}}} F x_{p} .
$$

The structure natural isomorphisms $s_{i} F_{*} \cong F_{*} s_{i}$ and $d_{i} F_{*} \cong F_{*} d_{i}$ are canonically obtained from the invertible structure constraints of the homomorphism, $\widehat{F}: 1_{F x_{i}} \cong F 1_{x_{i}}$ and $\hat{F}: F\left(u_{i}\right) \circ F\left(u_{i+1}\right) \cong F\left(u_{i} \circ u_{i+1}\right)$ (the commutativity of the needed six coherence diagrams in [25] is clear). Then, $F: \mathcal{B} \rightsquigarrow \mathcal{C}$ determines a pseudosimplicial functor, $\underline{\mathrm{N}} F: \underline{\mathrm{N}} \mathcal{B} \rightsquigarrow \underline{\mathrm{N} C}$, and therefore a functor $\int_{\Delta} F: \int_{\Delta} \mathcal{B} \rightarrow \int_{\Delta} \mathcal{C}$ and a corresponding map on the classifying spaces

$$
\mathrm{B} F: \mathrm{B} \mathcal{B} \rightarrow \mathrm{BC} .
$$

Thus, the classifying space construction $(26), \mathcal{C} \rightarrow \mathrm{BC}$, defines a functor from bicategories, with homomorphisms between them, to $\mathrm{CW}$-complexes. However, as we will see later, any lax, or oplax, functor $F: \mathcal{B} \rightsquigarrow \mathcal{C}$, that is, without the requirement that its structure constraints $\widehat{F}$ be isomorphisms, also induces a continuous map $\mathrm{B} F: \mathrm{B} \mathcal{B} \rightarrow \mathrm{BC}$, well defined up to homotopy equivalence.

\section{The simplicial sets geometric nerves of a bicategory}

The two isomorphic constructions (3) and (6) to define the nerve, and then the classifying space, of a category suggest different extensions to bicategories. Looking at (3), we were led to definition (21), which recovers the more traditional way through which a classifying space is assigned in the literature to certain kinds of bicategories, such as 2-categories (see Remark 3.2) or monoidal categories (see Remark 3.3). But the construction we have taken, in Definition 3.1, for the classifying space of a bicategory $\mathrm{BC}$ runs through the Grothendieck construction on its pseudosimplicial nerve, which implies that the cells of $\mathrm{BC}$ have little apparent intuitive connection with the cells of the original bicategory and that they do not enjoy any proper geometric meaning. However, looking at (6), we are led to dealing with another convincing way of associating a space to any bicategory $\mathcal{C}$ : through its unitary geometric nerve as defined by Street in [41] (see also Duskin [15] and Gurski [23]).

Definition 4.1 The unitary geometric nerve of a bicategory $\mathcal{C}$ is the simplicial set

$$
\Delta^{\mathrm{u}} \mathcal{C}: \Delta^{\mathrm{op}} \rightarrow \text { Set, }
$$

$$
[p] \mapsto \operatorname{NorLaxFunc}([p], \mathcal{C})
$$


whose $p$-simplices are the normal lax functors $F:[p] \rightsquigarrow \mathcal{C}$. If $a:[q] \rightarrow[p]$ is any map in $\Delta$, that is, a functor, the induced $a^{*}: \Delta^{\mathrm{u}} \mathcal{C}_{p} \rightarrow \Delta^{\mathrm{u}} \mathcal{C}_{q}$ carries $F:[p] \rightsquigarrow \mathcal{C}$ to the composite $F a:[q] \rightsquigarrow \mathcal{C}$, of $F$ with $a$.

This simplicial set $\Delta^{\mathrm{u}} \mathcal{C}$ encodes the entire bicategorical structure of $\mathcal{C}$, and the following lemma allows us to show a pleasing geometrical description of its simplices:

Lemma 4.2 Let $\mathcal{C}$ be a bicategory. Any system of data which consists of objects $F i, 0 \leq i \leq p$, morphisms $F_{i, j}: F j \rightarrow F i, 0 \leq i<j \leq p$, and deformations $\widehat{F}_{i, j, k}: F_{i, j} \circ F_{j, k} \Rightarrow F_{i, k}, 0 \leq i<j<k \leq p$, such that, for $0 \leq i<j<k<\ell \leq p$, the following square of deformations commutes in the category $\mathcal{C}(F \ell, F i)$,

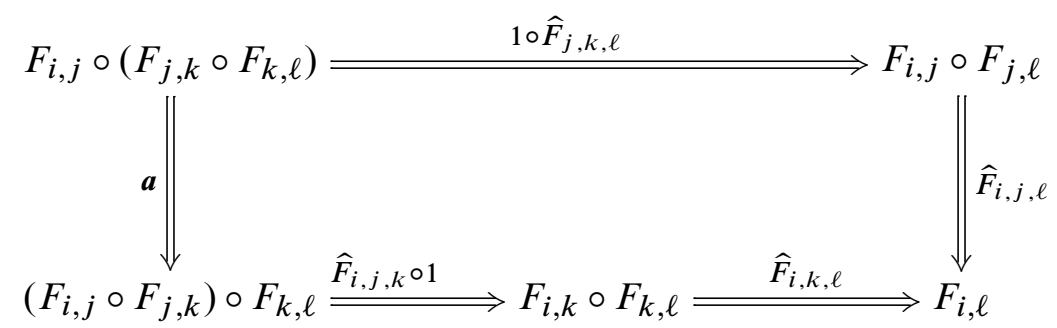

uniquely extends to a normal lax functor $F:[p] \rightsquigarrow \mathcal{C}$.

Proof The whole data for $F:[p] \rightsquigarrow \mathcal{C}$ are obtained by putting $F_{i, i}=1_{F i}, \widehat{F}_{i, i, j}=$ $\boldsymbol{l}: 1_{F i} \circ F_{i, j} \Rightarrow F_{i, j}, \widehat{F}_{i, j, j}=\boldsymbol{r}: F_{i, j} \circ 1_{F j} \Rightarrow F_{i, j}$, and $\widehat{F}_{i}=1_{1_{F i}}: 1_{F i} \Rightarrow 1_{F i}$. So defined, $F$ is actually a (normal) lax functor thanks to the commutativity of the coherence triangles in (14) and (16), and the equality in (15). The uniqueness of $F$ is clear.

Thus, for a bicategory $\mathcal{C}$, the vertices of its normal geometric nerve $\Delta^{\mathrm{u}} \mathcal{C}$ are the objects $F 0$ of $\mathcal{C}$, the 1 -simplices are the morphisms

$$
F 0 \stackrel{F_{0,1}}{\longleftarrow} F 1 \text {, }
$$

and the 2-simplices are triangles

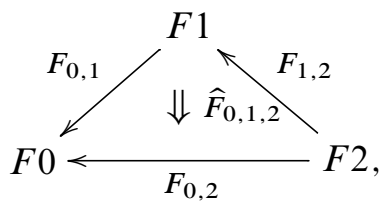


with $\widehat{F}_{0,1,2}: F_{0,1} \circ F_{1,2} \Rightarrow F_{0,2}$ a deformation in $\mathcal{C}$. For $p \geq 3$, a $p$-simplex of $\Delta^{\mathrm{u}} \mathcal{C}$ is geometrically represented by a diagram in $\mathcal{C}$ with the shape of the 2 -skeleton of an oriented standard $p$-simplex, whose faces are triangles

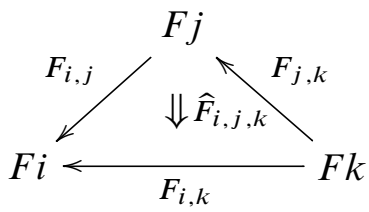

with objects $F i$ placed on the vertices, $0 \leq i \leq p$, morphisms $F_{i, j}: F j \rightarrow F i$ on the edges, $0 \leq i<j \leq p$, and $\widehat{F}_{i, j, k}: F_{i, j} \circ F_{j, k} \Rightarrow F_{i, k}$ deformations, for $0 \leq i<j<k \leq p$. These data are required to satisfy the condition that each tetrahedron

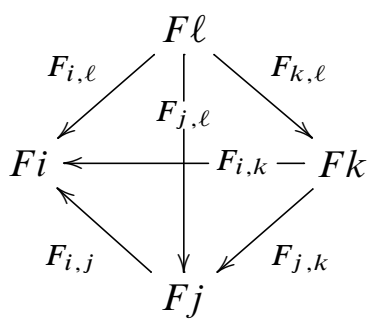

$$
\begin{gathered}
\widehat{F}_{i, j, k}: F_{i, j} \circ F_{j, k} \Rightarrow F_{i, k} \\
\widehat{F}_{i, j, \ell}: F_{i, j} \circ F_{j, \ell} \Rightarrow F_{i, \ell} \\
\widehat{F}_{i, k, \ell}: F_{i, k} \circ F_{k, \ell} \Rightarrow F_{i, \ell} \\
\widehat{F}_{j, k, \ell}: F_{j, k} \circ F_{k, \ell} \Rightarrow F_{j, \ell},
\end{gathered}
$$

for $0 \leq i<j<k<\ell \leq p$ is commutative in the sense that the following equation on deformations holds:
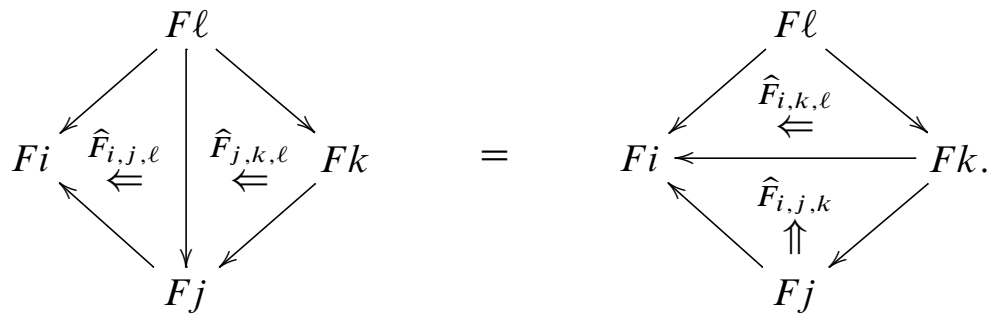

The simplicial set $\Delta^{\mathrm{u}} \mathcal{C}$ becomes coskeletal in dimensions greater than 3. More precisely, for $p \geq 3$, a $p$-simplex $F:[p] \rightsquigarrow \mathcal{C}$ of $\Delta^{\mathrm{u}} \mathcal{C}$ is determined uniquely by its boundary $\partial F=\left(d_{0} F, \ldots, d_{p} F\right)$

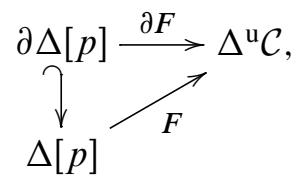

and, for $p \geq 4$, every possible boundary of a $p$-simplex, $\partial \Delta[p] \rightarrow \Delta^{\mathrm{u}} \mathcal{C}$, is actually the boundary $\partial F$ of a $p$-simplex $F$. 
For several discussions, it is suitable to handle the (nonnormalized) geometric nerve of a bicategory $\mathcal{C}$ :

Definition 4.3 The geometric nerve of a bicategory $\mathcal{C}$ is the simplicial set

$$
\begin{aligned}
\Delta \mathcal{C}: \quad \Delta^{\mathrm{op}} & \rightarrow \text { Set }, \\
& {[p] \mapsto \operatorname{LaxFunc}([p], \mathcal{C}), }
\end{aligned}
$$

that is, the simplicial set whose $p$-simplices are all lax functors $F:[p] \rightsquigarrow \mathcal{C}$.

Hence, the unitary geometric nerve $\Delta^{\mathrm{u}} \mathcal{C}$ becomes a simplicial subset of $\Delta \mathcal{C}$. The $p-$ simplices of the geometric nerve $\Delta \mathcal{C}$ are described similarly to those of the normalized one, but now they include deformations of $\mathcal{C}$

$$
\widehat{F}_{i}: 1_{F_{i}} \Rightarrow F_{i, i}, \quad 0 \leq i \leq p,
$$

with the requirement that the diagrams

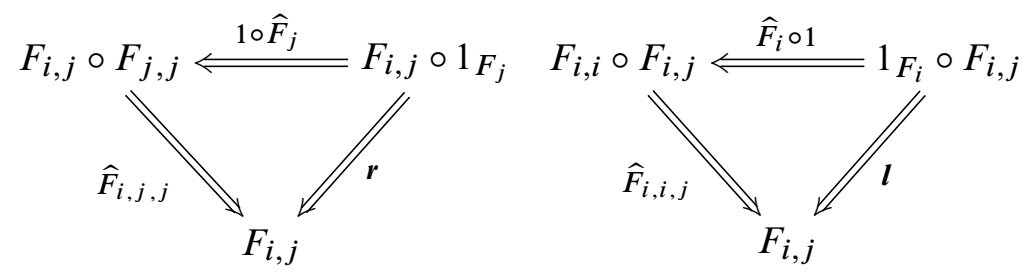

be commutative.

Note that the geometric nerve construction on bicategories, $\mathcal{C} \mapsto \Delta \mathcal{C}$, is clearly functorial on lax functors between bicategories, whereas $\Delta^{\mathrm{u}} \mathcal{C}$ is functorial only on normalized lax functors between bicategories.

We have used the above lax functors $[p] \rightsquigarrow \mathcal{C}$ to define geometric nerves of bicategories. However, there is no substantial reason for not considering oplax functors. In fact, we also have geometric nerves

$$
\begin{aligned}
\nabla \mathcal{C}: \quad \Delta^{\mathrm{op}} & \rightarrow \text { Set }, \\
{[p] } & \mapsto \text { OpLaxFunc }([p], \mathcal{C})
\end{aligned}
$$

that is, the simplicial set whose $p$-simplices are all oplax functors, $F:[p] \rightsquigarrow \mathcal{C}$, and

$$
\begin{aligned}
\nabla_{\mathrm{u}} \mathcal{C}: \quad \Delta^{\mathrm{op}} & \rightarrow \text { Set }, \\
& {[p] \mapsto \operatorname{NorOpLaxFunc}([p], \mathcal{C}) }
\end{aligned}
$$


the simplicial subset of $\nabla \mathcal{C}$, whose $p$-simplices are the normal oplax functors from $[p]$ to $\mathcal{C}$.

Remark 4.4 In [15], Duskin gave a characterization of the normal geometric nerve $\nabla_{\mathrm{u}} \mathcal{C}$ of a bicategory $\mathcal{C}$ in terms of its simplicial structure. The result states that a simplicial set is isomorphic to the normal geometric nerve of a bicategory if and only if it satisfies the coskeletal conditions above as well as supporting appropriate sets of "abstractly invertible" $1-$ and 2-simplices. For instance, recall that a bigroupoid is a bicategory $\mathcal{C}$ in which every deformation is invertible, that is, all categories $\mathcal{C}(y, x)$ are groupoids, and every morphism $u: y \rightarrow x$ is a biequivalence, that is, there exist a morphism $u^{\prime}: x \rightarrow y$ and deformations $u \circ u^{\prime} \Rightarrow 1_{y}$ and $1_{x} \Rightarrow u^{\prime} \circ u$. Then, the normal geometric nerve of a bicategory $\mathcal{C}$ becomes a Kan complex [33] (fibrant [19]), that is, every extension problem

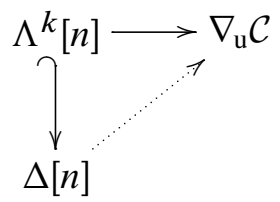

of any $(k, n)$-horn, $\Lambda^{k}[n] \rightarrow \nabla_{\mathrm{u}} \mathcal{C}$, has a solution if and only if the bicategory $\mathcal{C}$ is a bigroupoid. In such a case, the homotopy groups of $\nabla_{\mathrm{u}} \mathcal{C}$ are

$$
\begin{aligned}
& \pi_{i}\left(\nabla_{\mathrm{u}} \mathcal{C}, x\right) \\
& =\left\{\begin{array}{lll}
K_{0} \mathcal{C} & \text { the set of iso-classes of objects of } \mathcal{C} & \text { for } i=0, \\
K_{0} \mathcal{C}(x, x) & \text { the group of iso-classes of automorphisms in } \mathcal{C} \text { of } x & \text { for } i=1, \\
K_{1} \mathcal{C}(x, x) & \text { the abelian group of autodeformations in } \mathcal{C} \text { of } 1_{x} & \text { for } i=2, \\
0 & \text { for } i \geq 3 .
\end{array}\right.
\end{aligned}
$$

Furthermore, the normal geometric nerve construction embeds the category of bigroupoids, with normal homomorphisms between them, as a full and reflexive subcategory of the category of Kan complexes, whose replete image consists of those Kan complexes $K$ for which every $(k, n)$-horn $\Lambda^{k}[n] \rightarrow K$ has a unique filler

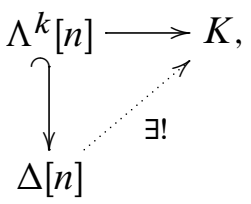

for all $0 \leq k \leq n$ and $n \geq 3$; that is, $K$ is a Kan 2-hypergroupoid in the sense of Duskin-Glenn (see Glenn [18] and Cegarra, Bullejos and Garzón [10], for example). 
Remark 4.5 The normal geometric nerve of a 2-category (even of an $n$-category) was first studied by Street [40]. In Worytkiewicz et al [46], but under the name of "2nerve of 2-categories", the normal geometric nerve construction $\Delta^{\mathrm{u}}$ was considered for proving that the category 2-Cat, of small 2-categories and 2-functors, has a Quillen model structure such that the functor $E x^{2} \Delta^{\mathrm{u}}:$ 2-Cat $\rightarrow$ Simpl.Set is a right Quillen equivalence of model categories, where $E x$ is the endofunctor in Simpl.Set right adjoint to the subdivision $S d$ (see Goerss and Jardine [19], for example). In Bullejos and Cegarra [6], it was proved that, for any 2-category $\mathcal{C}$, there is a natural homotopy equivalence $\mathrm{BC} \simeq \mathrm{B} \Delta^{\mathrm{u}} \mathcal{C}$, and therefore it follows that the correspondence $\mathcal{C} \mapsto \mathrm{BC}$ induces an equivalence between the corresponding homotopy category of 2-categories and the ordinary homotopy category of $\mathrm{CW}-$ complexes. By this correspondence, $2-$ groupoids correspond to spaces whose homotopy groups $\pi_{n}$ are trivial for $n>2$ [34], and from this point of view the use of 2-categories and their classifying spaces in homotopy theory goes back to Whitehead (1949) and Mac Lane-Whitehead (1950), since 2 -groupoids with only one object (2-groups) are the same as crossed modules. $\square$

Remark 4.6 Let $\mathcal{M}$ be a monoidal category. The delooping bicategory $\Omega^{-1} \mathcal{M}$ (see Remark 3.3) realizes the classifying space of the monoidal category and, in [7], it is shown how that realization can be made through the normal geometric nerve construction. That is, there is a homotopy equivalence $\mathrm{B} \Omega^{-1} \mathcal{M} \simeq \mathrm{B} \nabla_{\mathrm{u}} \Omega^{-1} \mathcal{M}$.

This geometric nerve $\nabla_{\mathrm{u}} \Omega^{-1} \mathcal{M}$ is a $3-$ coskeletal reduced (1-vertex) simplicial set, whose simplices have the following simplified interpretation: the 1 -simplices are the objects $F_{0,1}$ of $\mathcal{M}$, the 2 -simplices are morphisms of $\mathcal{M}$ of the form

$$
F_{0,2} \stackrel{F_{0,1,2}}{\longrightarrow} F_{0,1} \otimes F_{1,2}
$$

and the 3-simplices are commutative diagrams in $\mathcal{M}$ of the form

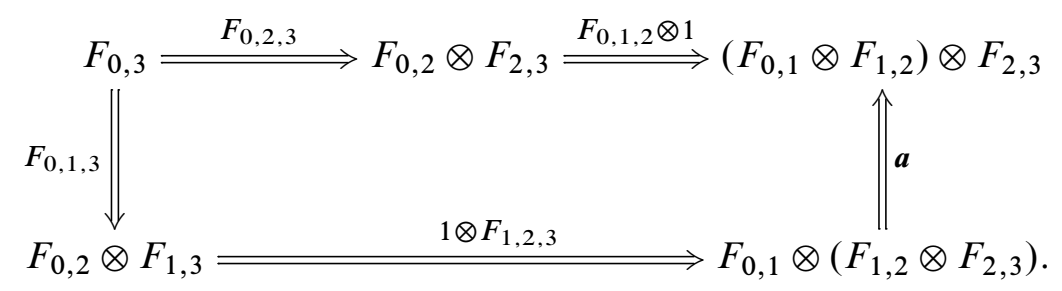

Bigroupoids with only one object are delooping bicategories $\Omega^{-1} \mathcal{M}$ of categorical groups [26] (Gr-categories [5]), that is, monoidal groupoids $\mathcal{M}$ in which translations are autoequivalences. Normal geometric nerves of categorical groups are studied in [8; $9 ; 11]$. 


\section{More Cat-valued nerves of a bicategory}

In this section, we shall describe the following commutative diagram with the various "nerves" of a bicategory $\mathcal{C}$ discussed in the paper:

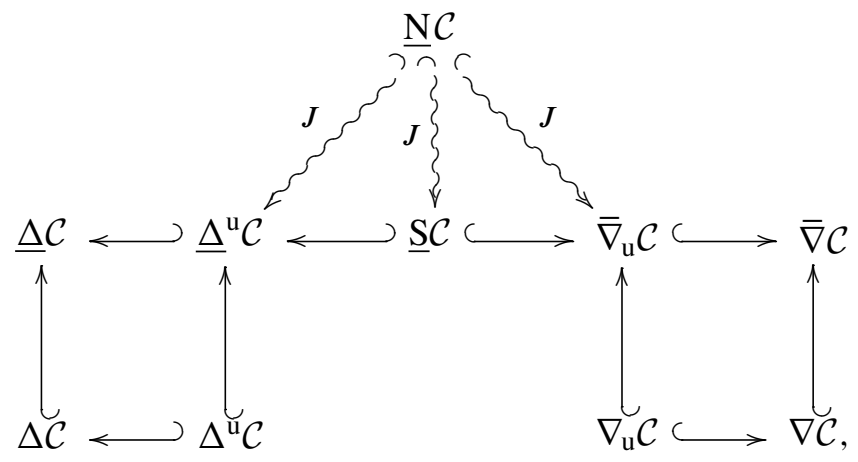

where $\underline{\mathrm{NC}}$, in the top row, is the pseudo simplicial nerve (21) of the bicategory, and $\Delta \mathcal{C}, \Delta^{\mathrm{u}} \mathcal{C}, \nabla \mathcal{C}$ and $\nabla_{\mathrm{u}} \mathcal{C}$, in the bottom row, are the geometric nerves of the bicategory, as defined in (32), (31), (35), and (36) respectively. In Definition 5.2 we introduce the remaining five Cat-valued nerves of bicategories. These associate to every bicategory $\mathcal{C}$ simplicial objects in Cat: Those called its unitary categorical geometric nerves, which are denoted by $\Delta^{\mathrm{u}} \mathcal{C}$ and $\bar{\nabla}_{\mathrm{u}} \mathcal{C}$, its categorical geometric nerves, denoted by $\underline{\Delta C}$ and $\bar{\nabla} \mathcal{C}$, and its Segal nerve, denoted by $\underline{S} \mathcal{C}$, respectively. As we will prove, each of these five simplicial categories can be thought of as a "rectification" of the pseudosimplicial nerve $\underline{N} \mathcal{C}(21)$ of the bicategory, since they model the same homotopy type as $\mathrm{BC}$. Furthermore, all these simplicial categories are "special" in the sense $[37 ; 43]$ that the Segal projection maps on them induce homotopy equivalences on classifying spaces; moreover, the latter, $\underline{S} \mathcal{C}$, is a weak 2-category in the sense of Tamsamani [42] and Simpson [38], that is, the Segal projection maps on it are surjective equivalences of categories [29] (or see the proof of Theorem 6.4 below). Unitary categorical geometric nerves for 2-categories were treated in [6] and for monoidal categories in [7]. Segal nerves of bicategories, also called 2-nerves in [29], enjoy many interesting properties whose study the cited paper by Lack and Paoli is mainly dedicated to.

Considering a lax functor $F:[p] \rightsquigarrow \mathcal{C}$ as a family of "paths" $F_{i, j}: F j \rightarrow F i$ between objects of the bicategory $\mathcal{C}$, and a lax transformation $\alpha: F \Rightarrow F^{\prime}$ as a "free homotopy" between them, it is natural to consider lax transformations relative to objects (ie, to end points) as those $\alpha$ that are constantly identities on objects, that is, with $\alpha i=1_{F_{i}}=1_{F_{i}^{\prime}}$, for all $0 \leq i \leq p$. This kind of lax transformations has been considered in [6], and also in $[28 ; 29 ; 17]$ under the name of icons (short for "identity component oplax natural transformations"). 
Remark 5.1 If $\mathcal{M}$ and $\mathcal{M}^{\prime}$ are monoidal categories, then a monoidal functor $F: \mathcal{M} \rightarrow$ $\mathcal{M}^{\prime}$ amounts precisely to a homomorphism between the corresponding delooping bicategories $\Omega^{-1} F: \Omega^{-1} \mathcal{M} \rightsquigarrow \Omega^{-1} \mathcal{M}^{\prime}$ (see Remark 3.3). If $F, F^{\prime}: \mathcal{M} \rightarrow \mathcal{M}^{\prime}$ are monoidal functors, then a monoidal transformation $\alpha: F \Rightarrow F^{\prime}$ is the same thing as a relative to objects lax transformation $\Omega^{-1} \alpha: \Omega^{-1} F \Rightarrow \Omega^{-1} F^{\prime}$. However, note that an arbitrary lax transformation $\beta: \Omega^{-1} F \Rightarrow \Omega^{-1} F^{\prime}$ consists of an object $x_{0}$ of $\mathcal{M}^{\prime} \quad(=\beta *$, the component at the unique object of $\Omega^{-1} \mathcal{M}$ ) and morphisms $\widehat{\beta}_{x}: x_{0} \otimes F x \rightarrow F^{\prime} x \otimes x_{0}$, one for each object $x$ of $\mathcal{M}$, satisfying the usual two conditions (see Section 2).

Definition 5.2 The categorical geometric nerves $\Delta \mathcal{C}$ and $\bar{\nabla} \mathcal{C}$ of a bicategory $\mathcal{C}$ are the simplicial categories

$$
\begin{aligned}
\underline{\Delta C}: \quad \Delta^{\mathrm{op}} & \rightarrow \text { Cat } \\
{[p] } & \mapsto \underline{\operatorname{LaxFunc}}([p], \mathcal{C}),
\end{aligned}
$$

whose category of $p$-simplices is the category of lax functors $F:[p] \rightsquigarrow \mathcal{C}$, with relative to objects lax transformations between them as arrows and

$$
\begin{aligned}
\bar{\nabla} \mathcal{C} \Delta^{\text {op }} & \rightarrow \text { Cat } \\
{[p] } & \mapsto \text { OpLaxFunc }([p], \mathcal{C}),
\end{aligned}
$$

whose category of $p$-simplices is the category of oplax functors $F:[p] \rightsquigarrow \mathcal{C}$, with relative to objects lax transformations between them as arrows, respectively.

The unitary categorical geometric nerves $\underline{\Delta}^{\mathrm{u}} \mathcal{C} \subseteq \underline{\Delta C}$ and $\bar{\nabla}_{\mathrm{u}} \mathcal{C} \subseteq \bar{\nabla} \mathcal{C}$ are the respective simplicial subcategories of normal lax functors and normal oplax functors $F:[p] \rightsquigarrow \mathcal{C}$, that is,

$$
\Delta^{\mathrm{u}} \mathcal{C}: \Delta^{\mathrm{op}} \rightarrow \text { Cat }
$$

$$
[p] \mapsto \underline{\operatorname{NorLaxFunc}}([p], \mathcal{C}),
$$

and

$$
\begin{aligned}
\bar{\nabla}_{\mathrm{u}} \mathcal{C}: \quad \Delta^{\mathrm{op}} & \rightarrow \text { Cat } \\
{[p] } & \mapsto \underline{\text { NorOpLaxFunc }}([p], \mathcal{C}) .
\end{aligned}
$$

The Segal nerve $\underline{S} \mathcal{C} \subseteq \underline{\Delta}^{\mathrm{u}} \mathcal{C}$ is the simplicial subcategory of normal homomorphisms $F:[p] \rightsquigarrow \mathcal{C}$, that is,

$$
\begin{aligned}
\underline{\text { SC}}: & \Delta^{\mathrm{op}} \rightarrow \text { Cat } \\
& {[p] \mapsto \underline{\operatorname{NorHom}}([p], \mathcal{C}), }
\end{aligned}
$$


Note that, for $F, F^{\prime}:[p] \rightsquigarrow \mathcal{C}$ two geometric $p$-simplices, the existence of morphisms $\hat{\alpha}: F \Rightarrow F^{\prime}$ in the categorical geometric nerve requires that $F i=F^{\prime} i$ for all $0 \leq i \leq p$, and, in such a case, an $\hat{\alpha}: F \Rightarrow F^{\prime}$ consists of a family of deformations in $\mathcal{C}$

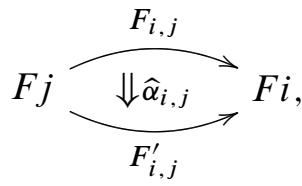

for $0 \leq i \leq j \leq p$, such that
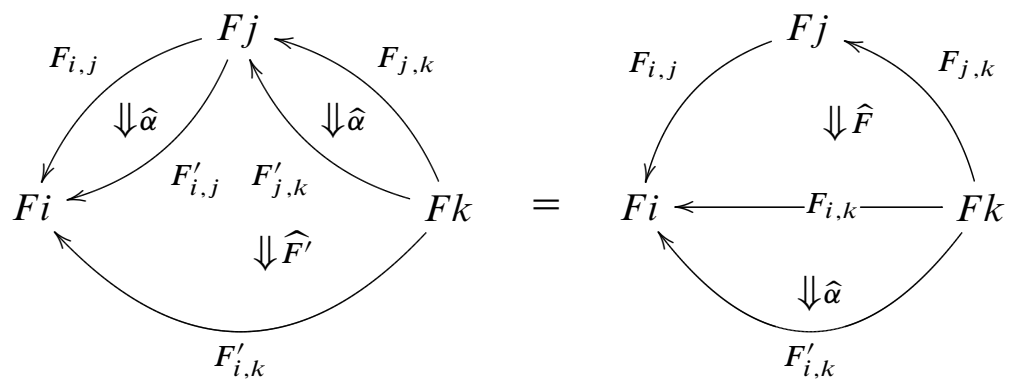

for $0 \leq i \leq j \leq k \leq p$, and, for $0 \leq i \leq p$,
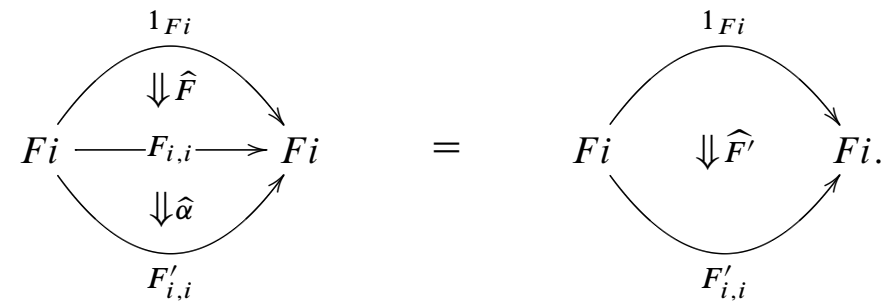

We now complete the description of diagram (37):

Definition 5.3 (i) The simplicial maps $\Delta^{\mathrm{u}} \mathcal{C} \hookrightarrow \Delta \mathcal{C}$ and $\nabla_{\mathrm{u}} \mathcal{C} \hookrightarrow \nabla \mathcal{C}$ are inclusions.

(ii) The simplicial functors $\underline{S} \mathcal{C} \hookrightarrow \underline{\Delta}^{\mathrm{u}} \mathcal{C} \hookrightarrow \underline{\Delta C}$ and $\bar{\nabla}_{\mathrm{u}} \mathcal{C} \hookrightarrow \bar{\nabla} \mathcal{C}$ are all inclusions.

(iii) The simplicial functor $\underline{S} \mathcal{C} \hookrightarrow \bar{\nabla}_{\mathrm{u}} \mathcal{C}$ is the full embedding defined by

$$
\left\{\begin{array}{l}
(F, \widehat{F}) \stackrel{\mapsto}{ }\left(F, \widehat{F}^{-1}\right), \\
(F, \widehat{F}) \stackrel{\widehat{\alpha}}{\Longrightarrow}\left(F^{\prime}, \widehat{F}^{\prime}\right) \mapsto\left(F, \widehat{F}^{-1}\right) \stackrel{\widehat{\alpha}}{\Longrightarrow}\left(F^{\prime}, \widehat{F}^{\prime}-1\right) .
\end{array}\right.
$$


(iv) Each geometric nerve is regarded as a discrete simplicial category (ie, with only identities) and the simplicial functors

$$
\Delta \mathcal{C} \hookrightarrow \Delta \underline{C}, \quad \Delta^{\mathrm{u}} \mathcal{C} \hookrightarrow \underline{\Delta}^{\mathrm{u}} \mathcal{C}, \quad \nabla \mathcal{C} \hookrightarrow \bar{\nabla} \mathcal{C}, \quad \nabla_{\mathrm{u}} \mathcal{C} \hookrightarrow \bar{\nabla}_{\mathrm{u}} \mathcal{C}
$$

are all inclusions, as each geometric nerve is the simplicial set of objects of the corresponding categorical geometric nerve.

(v) The pseudosimplicial functor $J: \underline{N} \mathcal{C} \rightsquigarrow \underline{S} \mathcal{C}$ is defined as follows:

First, note that we have the equalities

$$
\begin{aligned}
& \underline{\mathrm{S}}_{0}=\mathrm{Ob} \mathcal{C}=\underline{\mathrm{NC}}_{0}, \\
& \underline{\mathrm{S}}_{1}=\bigsqcup_{x_{0}, x_{1}} \mathcal{C}\left(x_{1}, x_{0}\right)=\underline{\mathrm{NC}}_{1}
\end{aligned}
$$

by identifying a normal homomorphism $F:[0] \rightsquigarrow \mathcal{C}$ with the object $F 0$ and a normal homomorphism $F$ : [1] $\rightsquigarrow \mathcal{C}$ with the arrow $F_{0,1}: F 1 \rightarrow F 0$ (see Lemma 4.2). We then take $J_{n}: \underline{\mathrm{N}} \mathcal{C}_{n} \rightarrow \underline{\mathrm{S}} \mathcal{C}_{n}$ to be the identity functor for $n=0,1$.

For each $n \geq 2$, let

$$
J_{n}: \underline{\mathrm{NC}}_{n} \hookrightarrow \underline{\mathrm{S}}_{n}
$$

be the functor taking an object of

$$
\underline{\mathrm{N}}_{n}=\bigsqcup_{\left(x_{0}, \ldots, x_{n}\right) \in \mathrm{Ob} \mathcal{C}^{n+1}} \mathcal{C}\left(x_{1}, x_{0}\right) \times \cdots \times \mathcal{C}\left(x_{n}, x_{n-1}\right),
$$

say the one given by the string

$$
F 0 \stackrel{F_{0,1}}{\longleftarrow} F 1 \stackrel{F_{1,2}}{\longleftarrow} \cdots \stackrel{F_{n-1, n}}{\longleftarrow} F n
$$

to the normal geometric $n$-simplex of $\mathcal{C}$

$$
F^{\mathrm{e}}=\left(F^{\mathrm{e}}, \hat{F}^{\mathrm{e}}\right):[n] \rightsquigarrow \mathcal{C},
$$

which places the objects $F^{\mathrm{e}} i=F i$ at the vertices and the morphism $F_{i, i+1}^{\mathrm{e}}=$ $F_{i, i+1}: F_{i+1} \rightarrow F_{i}$ at the edges $i+1 \rightarrow i$. For $0 \leq i<j<n$, the arrows $F_{i, j+1}^{\mathrm{e}}: F_{j+1} \rightarrow F_{i}$ are then inductively given by

$$
F_{i, j+1}^{\mathrm{e}}=F_{i, j}^{\mathrm{e}} \circ F_{j, j+1},
$$


so that the triangles

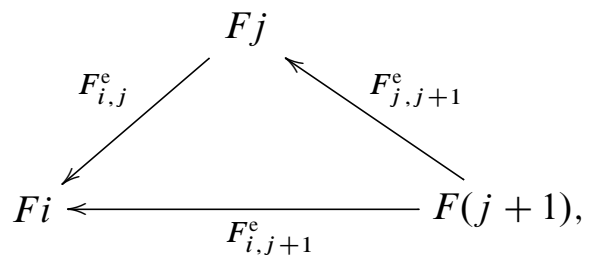

commute and the deformations $\hat{F}_{i, j, j+1}^{\mathrm{e}}: F_{i, j}^{\mathrm{e}} \circ F_{j, j+1}^{\mathrm{e}} \Rightarrow F_{i, j+1}^{\mathrm{e}}$ are all taken to be identities. For $0 \leq i<j<k<n$, the $2-$ cells $\widehat{F}_{i, j, k+1}^{\mathrm{e}}: F_{i, j}^{\mathrm{e}} \circ F_{j, k+1}^{\mathrm{e}} \Rightarrow F_{i, k+1}^{\mathrm{e}}$ are canonically determined by the associativity constraints of $\mathcal{C}$ through the commutative diagrams

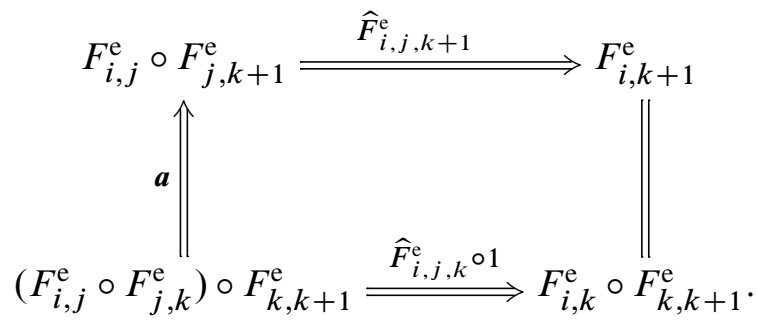

Thus, for example:

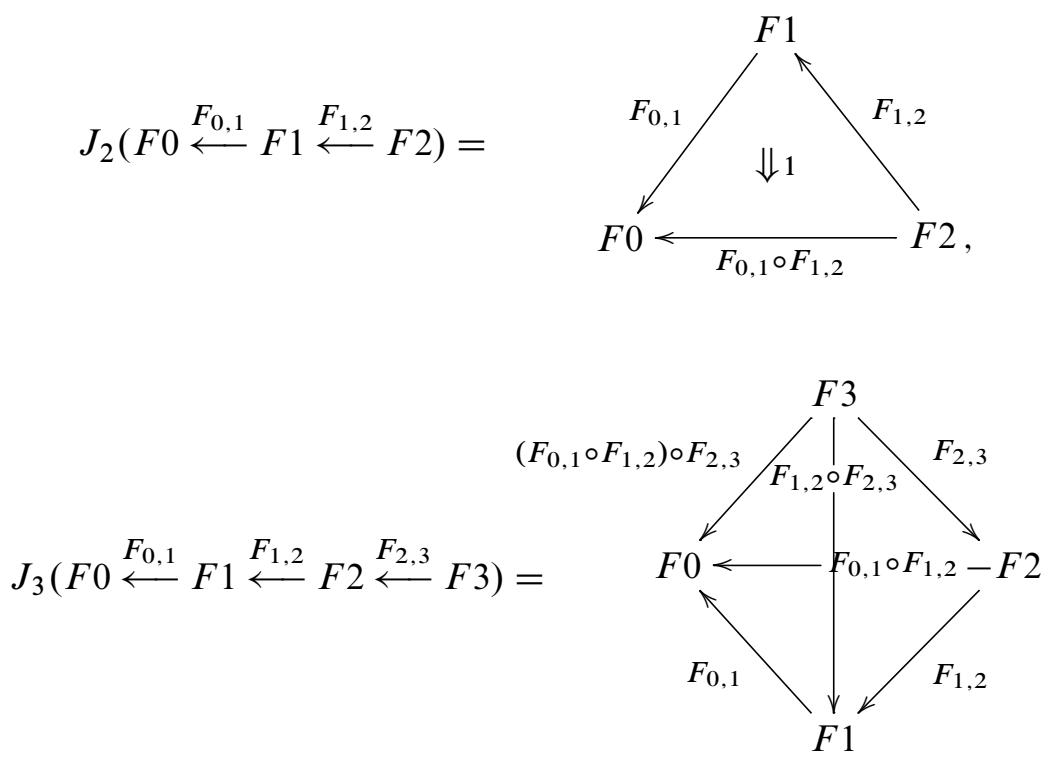


with the deformations

$$
\begin{aligned}
& \widehat{F}_{0,1,2}^{\mathrm{e}}=1: F_{0,1} \circ F_{1,2} \Rightarrow F_{0,1} \circ F_{1,2} \\
& \widehat{F}_{0,1,3}^{\mathrm{e}}=\boldsymbol{a}^{-1}: F_{0,1} \circ\left(F_{1,2} \circ F_{2,3}\right) \Rightarrow\left(F_{0,1} \circ F_{1,2}\right) \circ F_{2,3} \\
& \widehat{F}_{0,2,3}^{\mathrm{e}}=1:\left(F_{0,1} \circ F_{1,2}\right) \circ F_{2,3} \Rightarrow\left(F_{0,1} \circ F_{1,2}\right) \circ F_{2,3} \\
& \widehat{F}_{1,2,3}^{\mathrm{e}}=1: F_{1,2} \circ F_{2,3} \Rightarrow F_{1,2} \circ F_{2,3},
\end{aligned}
$$

and so on.

It is a consequence of the coherence theorem for bicategories that each

$$
J_{n}\left(F_{0} \stackrel{F_{0,1}}{\longleftarrow} F_{1} \stackrel{F_{1,2}}{\longleftarrow} \ldots \stackrel{F_{n-1, n}}{\longleftarrow} F_{n}\right)=\left(F^{\mathrm{e}}, \widehat{F}^{\mathrm{e}}\right):[n] \rightsquigarrow \mathcal{C}
$$

is in fact a normal geometric $n$-simplex of $\mathcal{C}$ (recall Lemma 4.2). Indeed, since every deformation $\hat{F}_{i, j, k}^{\mathrm{e}}: F_{i, j}^{\mathrm{e}} \circ F_{j, k}^{\mathrm{e}} \Rightarrow F_{i, k}$ is invertible, it is actually a normal homomorphism $F^{\mathrm{e}}:[n] \rightsquigarrow \mathcal{C}$.

Further, the functor $J_{n}$ on an arrow

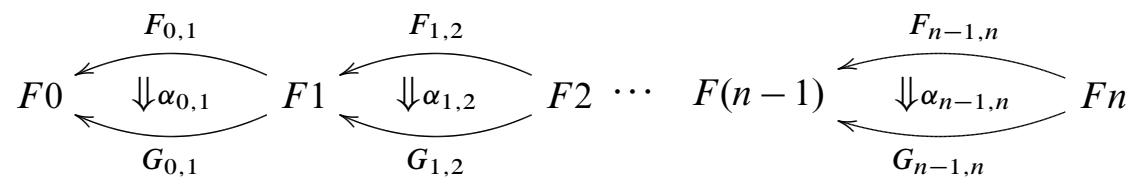

in $\underline{\mathrm{N}} \mathcal{C}_{n}$ is the arrow in $\underline{\mathrm{S}} \mathcal{C}_{n}$ (ie, the relative to objects lax transformation) $\hat{\alpha}^{\mathrm{e}}: F^{\mathrm{e}} \Rightarrow G^{\mathrm{e}}$, as in (43), inductively given by the deformations

$$
\hat{\alpha}_{i, j+1}^{\mathrm{e}}= \begin{cases}\alpha_{i, i+1} & \text { if } j=i, \\ \hat{\alpha}_{i, j}^{\mathrm{e}} \circ \alpha_{j, j+1} & \text { if } j>i .\end{cases}
$$

From its construction, it is clear that each $J_{n}: \underline{\mathrm{N}} \mathcal{C}_{n} \rightarrow \underline{\mathrm{S}} \mathcal{C}_{n}$ is a faithful and injective on objects functor. That $J_{n}$ is full follows from the equalities (44) since every geometric simplex $F:[n] \rightsquigarrow \mathcal{C}$ in $\underline{S} \mathcal{C}$ is a homomorphism, that is, the deformations $\widehat{F}_{i, j, k}$ are all invertible.

The family of functors $J_{n}: \underline{\mathrm{N}} \mathcal{C}_{n} \rightarrow \underline{\mathrm{S}} \mathcal{C}_{n}$, together with the natural isomorphisms $J_{n-1} d_{i} \cong d_{i} J_{n}$ and $J_{n} s_{i} \cong s_{i} J_{n-1}$, canonically induced by the associativity and unit constraints $\boldsymbol{a}, \boldsymbol{l}$, and $\boldsymbol{r}$ of the bicategory $\mathcal{C}$, becomes a morphism of supercoherent structures [25], and this defines our pseudosimplicial full embedding $J: \underline{N} \mathcal{C} \rightsquigarrow \underline{S} \mathcal{C}$. Actually, $J$ is a pseudo equivalence, as we will show later in the proof of Theorem 6.4.

To end this section, we stress that the construction $\underline{N}$ is functorial on homomorphisms between bicategories $\mathcal{C} \rightsquigarrow \mathcal{C}^{\prime}$, whereas $\underline{S} \mathcal{C}$ is on normal homomorphisms, $\Delta^{\mathrm{u}} \mathcal{C}$ and 
$\underline{\Delta}^{\mathrm{u}} \mathcal{C}$ are on normal lax functors, $\Delta \mathcal{C}$ and $\underline{\Delta} \mathcal{C}$ are on lax functors, $\nabla_{\mathrm{u}} \mathcal{C}$ and $\bar{\nabla}_{\mathrm{u}} \mathcal{C}$ are on normal oplax functors, and $\nabla \mathcal{C}$ and $\bar{\nabla} \mathcal{C}$ are on all oplax functors.

\section{The homotopy invariance theorem}

In this section, our goal is to prove the following:

Theorem 6.1 For any bicategory $\mathcal{C}$, all the maps in the diagram

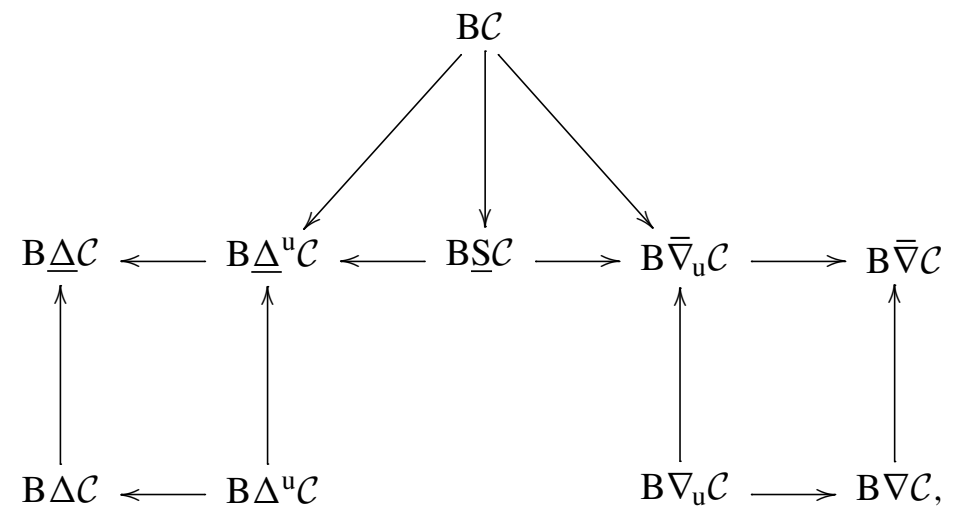

induced by (37) on classifying spaces, are homotopy equivalences.

By symmetry, we only will attack the problem of proving what concerns the maps in the middle and on the left of the diagram. The other homotopy equivalences are proven in a parallel way. The proof falls naturally apart in four theorems as below.

Theorem 6.2 For any bicategory $\mathcal{C}$, both simplicial inclusion functors $\Delta \mathcal{C} \hookrightarrow \Delta \mathcal{C}$ and $\Delta^{\mathrm{u}} \mathcal{C} \hookrightarrow{ }^{\mathrm{u}} \mathcal{C}$ induce homotopy equivalences on the corresponding classifying spaces, thus $\mathrm{B} \Delta \mathcal{C} \simeq \mathrm{B} \underline{A C}$ and $\mathrm{B} \Delta^{\mathrm{u}} \mathcal{C} \simeq \mathrm{B} \underline{\Delta}^{\mathrm{u}} \mathcal{C}$.

Proof The following argument is for $\Delta \mathcal{C} \hookrightarrow \underline{\mathcal{C}}$, and we leave to the reader to check that all the following constructions below restrict to the subinclusion $\Delta^{\mathrm{u}} \mathcal{C} \hookrightarrow \underline{\Delta}^{\mathrm{u}} \mathcal{C}$, by giving so a corresponding proof for this latter.

Let $\mathrm{N} \underline{\Delta} \mathcal{C}$ be the bisimplicial set obtained from the simplicial category $\underline{\Delta C}$ by composing with the nerve of categories functor (3). Then, a $(p, q)$-simplex of $\mathrm{N} \underline{\Delta C}$ is a string

$$
\xi=\left(F^{0} \stackrel{\widehat{\alpha}^{1}}{\Longleftarrow} F^{1} \stackrel{\widehat{\alpha}^{2}}{\Longleftarrow} \cdots \stackrel{\widehat{\alpha}^{p}}{\Longleftarrow} F^{p}\right)
$$


of $p$ composable arrows in the category $\underline{\Delta} \mathcal{C}_{q}=\underline{\operatorname{LaxFunc}}([q], \mathcal{C})$, of lax functors $[q] \rightsquigarrow \mathcal{C}$. The vertical face and degeneracy operators in $\mathrm{N} \Delta \mathcal{C}$

$$
\mathrm{N} \underline{\Delta C}_{p, q+1} \stackrel{s_{m}^{v}}{\longleftarrow} \mathrm{N} \underline{\Delta C}_{p, q} \stackrel{d_{m}^{v}}{\longrightarrow} \mathrm{N} \underline{\Delta C}_{p, q-1}, \quad 0 \leq m \leq q,
$$

are induced by those of $\underline{\Delta \mathcal{C}}$, that is,

$$
d_{m}^{v} \xi=\left(d_{m} F^{0} \stackrel{d_{m} \widehat{\alpha}^{1}}{\Longleftarrow} d_{m} F^{1} \stackrel{d_{m} \widehat{\alpha}^{2}}{\Longleftarrow} \cdots \stackrel{d_{m} \widehat{\alpha}^{p}}{\Longleftarrow} d_{m} F^{p}\right),
$$

and similarly $s_{m}^{v} \xi$; whereas the horizontal ones

$$
\mathrm{N} \underline{\Delta} \mathcal{C}_{p+1, q} \stackrel{s_{m}^{h}}{\longleftarrow} \mathrm{N} \underline{\Delta} \mathcal{C}_{p, q} \stackrel{d_{m}^{h}}{\longrightarrow} \mathrm{N} \underline{\Delta C}_{p-1, q}, 0 \leq m \leq p,
$$

are those of $\mathrm{N}\left(\underline{\Delta} \mathcal{C}_{q}\right)$, which are given by the formulas (5).

Since $\Delta \mathcal{C}$ is a discrete simplicial category, $\mathrm{N} \Delta \mathcal{C}$ is a bisimplicial set that is constant in the horizontal direction. The induced bisimplicial inclusion $\mathrm{N} \Delta \mathcal{C} \rightarrow \mathrm{N} \Delta \mathcal{C}$ is then, for each $p \geq 0$, the composite simplicial map

$$
\begin{aligned}
& \Delta \mathcal{C}=\mathrm{N} \underline{\Delta} \mathcal{C}_{0, *} \stackrel{s_{0}^{h}}{\hookrightarrow} \mathrm{N} \underline{\Delta} \mathcal{C}_{1, *} \stackrel{s_{0}^{h}}{\hookrightarrow} \ldots \stackrel{s_{0}^{h}}{\hookrightarrow} \mathrm{N} \underline{\Delta} \mathcal{C}_{p, *} . \\
& F \longmapsto(F \stackrel{\hat{1}}{\Longleftarrow} F \stackrel{\hat{1}}{\Longleftarrow}(\underline{p} \stackrel{\hat{1}}{\Longleftarrow} F)
\end{aligned}
$$

Taking into account now the homeomorphisms (9), to prove that $\mathrm{B} \Delta \mathcal{C} \rightarrow \mathrm{B} \Delta \mathcal{C}$ is a homotopy equivalence, we are going to prove that the induced simplicial map on diagonals $\Delta \mathcal{C} \rightarrow \operatorname{diagN} \underline{\underline{C}}$ is a weak equivalence. For it, as every pointwise weak homotopy equivalence bisimplicial map is a diagonal weak homotopy equivalence [19, IV, Proposition 1.7], it suffices to prove that every each of these simplicial maps (51) is a weak homotopy equivalence. Actually, we will prove more: Every simplicial map

$$
\begin{aligned}
s_{0}^{h}: & \mathrm{N} \Delta \mathcal{C}_{p, *} \hookrightarrow \mathrm{N} \underline{\Delta} \mathcal{C}_{p+1, *}, \\
& \left(F^{0} \stackrel{\hat{\alpha}^{1}}{\Longleftarrow} F^{1} \stackrel{\widehat{\alpha}^{2}}{\Longleftarrow} \cdots \stackrel{\hat{\alpha}^{p}}{\Longleftarrow} F^{p}\right) \mapsto\left(F^{0} \stackrel{\hat{1}}{\Longleftarrow} F^{0} \stackrel{\hat{\alpha}^{1}}{\Longleftarrow} F^{1} \stackrel{\widehat{\alpha}^{2}}{\Longleftarrow} \cdots \hat{\alpha}^{p} F^{p}\right)
\end{aligned}
$$

embeds the simplicial set $\mathrm{N} \underline{\Delta} \mathcal{C}_{p, *}$ into $\mathrm{N} \underline{\Delta} \mathcal{C}_{p+1, *}$ as a simplicial deformation retract. To do so, since $d_{0}^{h} s_{0}^{h}=1$, it is enough to exhibit a simplicial homotopy

$$
H^{p}: 1 \Rightarrow s_{0}^{h} d_{0}^{h}: \mathrm{N} \underline{\Delta} \mathcal{C}_{p, *} \rightarrow \mathrm{N} \underline{\Delta} \mathcal{C}_{p, *},
$$

for each $p \geq 1$. 
We first consider the case $p=1$. Then, to define $H^{1}$, we begin by constructing a simplicial homotopy

$$
T: d_{1}^{h} \Rightarrow d_{0}^{h}: \mathrm{N} \underline{\Delta} \mathcal{C}_{1, *} \rightarrow \mathrm{N} \underline{\Delta} \mathcal{C}_{0, *}=\Delta \mathcal{C},
$$

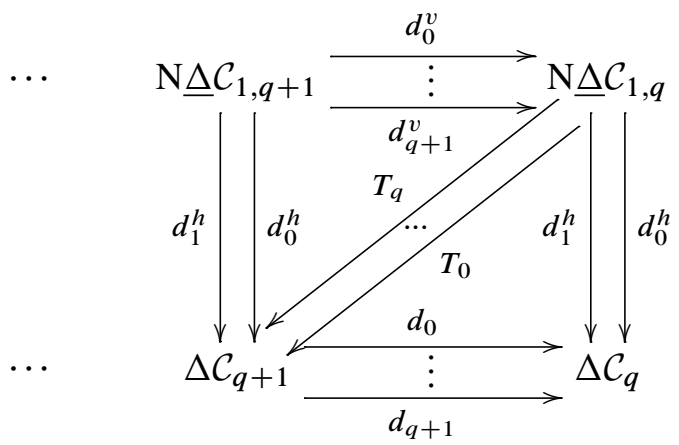

by the maps $T_{m}: \mathrm{N} \Delta \mathcal{C}_{1, q} \rightarrow \Delta \mathcal{C}_{q+1}, 0 \leq m \leq q$, defined as follows: For any arrow $\hat{\alpha}: F^{1} \Rightarrow F^{0}$ in the category $\underline{\Delta} \mathcal{C}_{q}=\underline{\operatorname{LaxFunc}}([q], \mathcal{C})$, and writing $F^{1} j=F^{0} j$ by $F j$ for $0 \leq j \leq q$, then

$$
T_{m}(\hat{\alpha}):[q+1] \rightsquigarrow \mathcal{C}
$$

is the geometric $(q+1)$-simplex of $\mathcal{C}$ that places

- at each vertex $i, 0 \leq i \leq q+1$, the object

$$
\begin{cases}F i & \text { if } i \leq m, \\ F(i-1) & \text { if } i>m ;\end{cases}
$$

- at each edge $j \rightarrow i, 0 \leq i \leq j \leq q+1$, the arrow

$$
\begin{cases}F_{i, j}^{1}: F j \rightarrow F i & \text { if } j \leq m, \\ F_{i, j-1}^{0}: F(j-1) \rightarrow F i & \text { if } i \leq m<j, \\ F_{i-1, j-1}^{0}: F(j-1) \rightarrow F(i-1) & \text { if } m<i\end{cases}
$$

- at each triangle

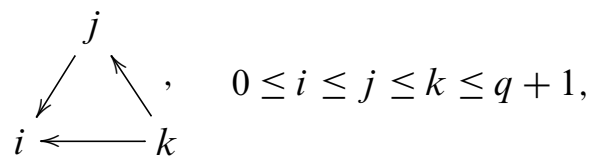


the 2 -cell in $\mathcal{C}$

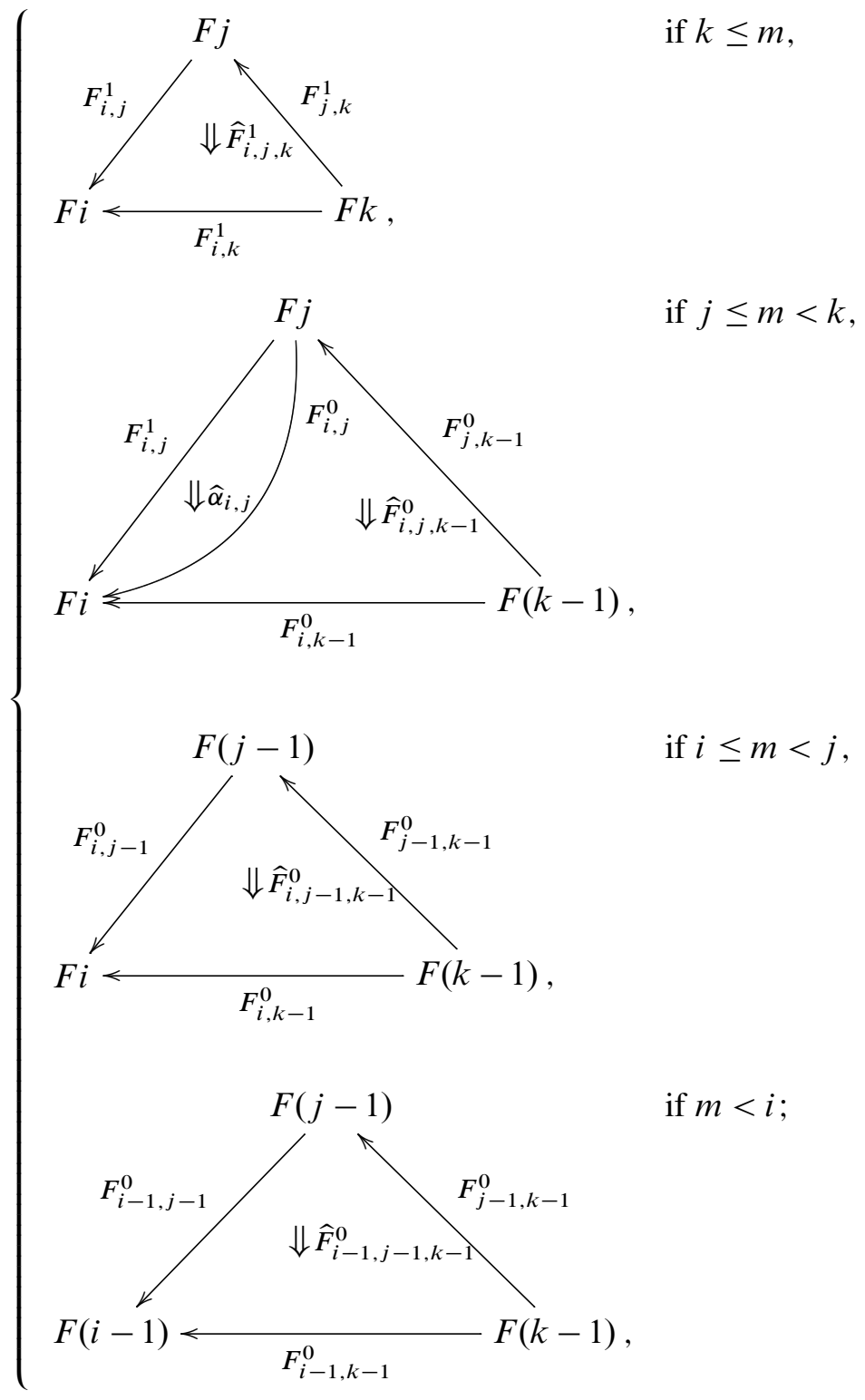

and whose unit structure deformations (33) are

$$
\begin{cases}\widehat{F}_{i}^{1}: 1_{F i} \Rightarrow F_{i, i}^{1}, & \text { if } i \leq m, \\ \widehat{F}_{i-1}^{0}: 1_{F(i-1)} \Rightarrow F_{i-1, i-1}^{0}, & \text { if } m<i\end{cases}
$$


It is straightforward to verify that the above definitions do indeed give the simplicial homotopy (53) as predicted. Further, note that we have the equalities $T_{0}=s_{0} d_{1}^{h}$ and $T_{m} s_{0}^{h}=s_{m}$, for all $0 \leq m \leq q$.

Then, the simplicial homotopy $H^{1}: 1 \Rightarrow s_{0}^{h} d_{0}^{h}$

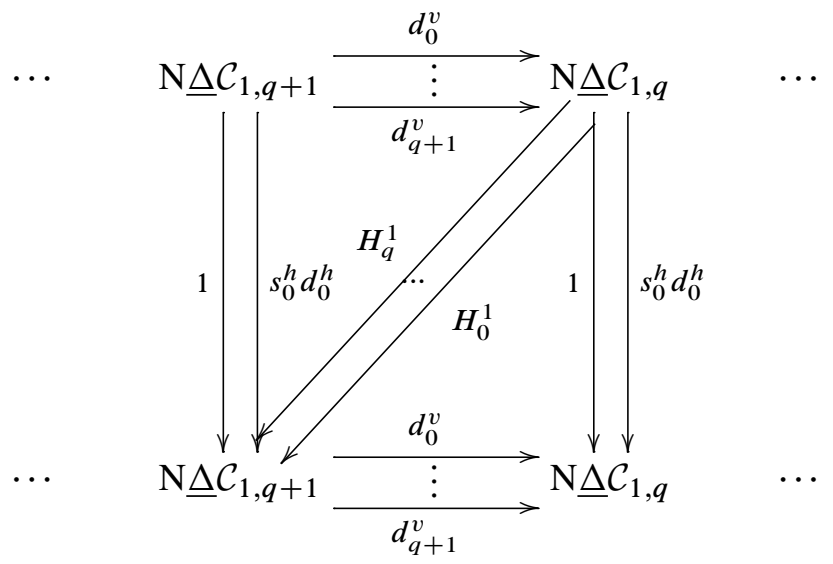

is defined by the maps $H_{m}^{1}, 0 \leq m \leq q$, as above, which apply an arrow $\widehat{\alpha}: F^{1} \Rightarrow F^{0}$ in $\mathrm{N} \Delta \mathcal{C}_{q}$ to the arrow

$$
s_{m} F^{1} \stackrel{H_{m}^{1}(\widehat{\alpha})}{\Longrightarrow} T_{m}(\widehat{\alpha})
$$

in $\mathrm{N} \Delta \mathcal{C}_{q+1}$, given by the family of deformations of $\mathcal{C}$

$$
H_{m}^{1}(\widehat{\alpha})_{i, j}= \begin{cases}1: F_{i, j}^{1} \Rightarrow F_{i, j}^{1} & \text { if } j \leq m, \\ \alpha_{i, j-1}: F_{i, j-1}^{1} \Rightarrow F_{i, j-1}^{0} & \text { if } i \leq m<j, \\ \alpha_{i-1, j-1}: F_{i-1, j-1}^{1} \Rightarrow F_{i-1, j-1}^{0} & \text { if } i<m\end{cases}
$$

for $0 \leq i \leq j \leq q+1$.

One easily observes that $H_{0}^{1}(\widehat{\alpha})=s_{0}^{v}(\widehat{\alpha})$ and $d_{q+1}^{v} H_{q}^{1}(\widehat{\alpha})=1_{F^{1}}$, whence $d_{0}^{v} H_{0}^{1}=1$ and $d_{q+1}^{v} H_{q}^{1}=s_{0}^{h} d_{0}^{h}$. Checking in full the remaining homotopy simplicial identities needed for $H^{1}: 1 \Rightarrow s_{0}^{h} d_{0}^{h}$ to be a simplicial homotopy is again straightforward (though quite tedious) and we will leave it to the reader. 
Finally, for an arbitrary $p \geq 2$, the simplicial homotopy $H^{p}: 1 \Rightarrow s_{0}^{h} d_{0}^{h}$

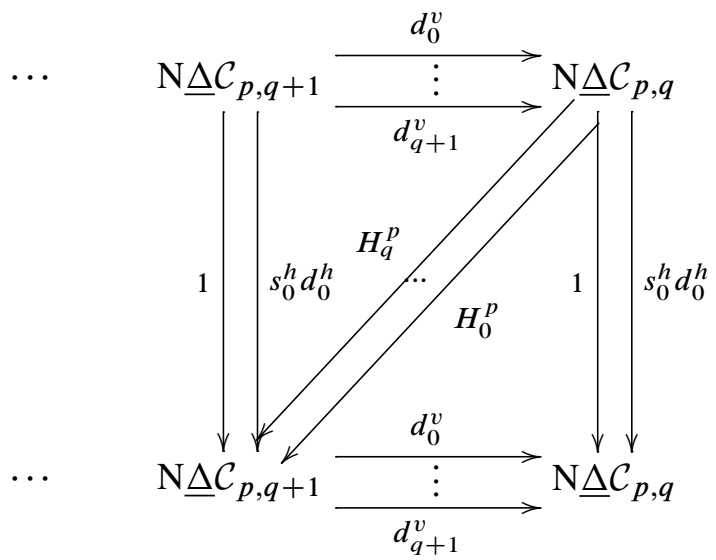

is given by the maps $H_{m}^{p}, 0 \leq m \leq q$, which take a $(p, q)$-simplex of $\mathrm{N} \underline{\Delta} \mathcal{C}$, say

$$
\xi=\left(F^{0} \stackrel{\hat{\alpha}^{1}}{\Longleftarrow} F^{1} \stackrel{\hat{\alpha}^{2}}{\Longleftarrow} \cdots \stackrel{\hat{\alpha}^{p}}{\Longleftarrow} F^{p}\right),
$$

to the $(p, q+1)$-simplex

$$
H_{m}^{p}(\xi)=\left(T_{m}\left(\hat{\alpha}^{1}\right) \stackrel{H_{m}^{1}\left(\widehat{\alpha}^{1}\right)}{\Longleftarrow} s_{m} F^{1} \stackrel{s_{m} \widehat{\alpha}^{2}}{\Longleftarrow} s_{m} F^{2} \ldots \stackrel{s_{m} \widehat{\alpha}^{p}}{\Longleftarrow} s_{m} F^{p}\right),
$$

where $H_{m}^{1}\left(\hat{\alpha}^{1}\right)$ is as in (54). The simplicial identities that make $H^{p}$ a simplicial homotopy are at this stage much easier to verify. This completes the proof.

The proof of the following theorem involves the "normalization" process.

Theorem 6.3 For any bicategory $\mathcal{C}$, both the simplicial inclusion functor $\underline{\Delta}^{\mathrm{u}} \mathcal{C} \hookrightarrow \underline{\mathcal{C}}$ and the simplicial inclusion map $\Delta^{\mathrm{u}} \mathcal{C} \hookrightarrow \Delta \mathcal{C}$ induce homotopy equivalences on the corresponding classifying spaces, thus $\mathrm{B} \underline{\Delta}^{\mathrm{u}} \mathcal{C} \simeq \mathrm{B} \underline{\mathcal{C}}$ and $\mathrm{B} \Delta^{\mathrm{u}} \mathcal{C} \simeq \mathrm{B} \Delta \mathcal{C}$.

Proof Since we have the commutative square

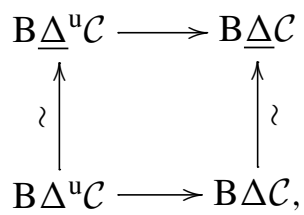

in which, by Theorem 6.3 above, both vertical maps are homotopy equivalences, it is sufficient to prove that the simplicial functor $\underline{\Delta}^{\mathrm{u}} \mathcal{C} \hookrightarrow \underline{\Delta} \mathcal{C}$ induces a homotopy equivalence on classifying spaces. 
We will prove that, for each $p \geq 0$, the inclusion functor

$$
\underline{\Delta}^{\mathrm{u}} \mathcal{C}_{p}=\underline{\operatorname{NorLaxFunc}}([p], \mathcal{C}) \hookrightarrow \underline{\Delta \mathcal{C}_{p}}=\underline{\operatorname{LaxFunc}}([p], \mathcal{C})
$$

has a right adjoint. Then, every induced map $\mathrm{B} \underline{\Delta}^{\mathrm{u}} \mathcal{C}_{p} \rightarrow \mathrm{B} \underline{\Delta} \mathcal{C}_{p}$ is a homotopy equivalence and the result follows from Theorem 2.2.

The right adjoint functor $(-)^{\mathrm{u}}: \underline{\Delta} \mathcal{C}_{p} \rightarrow \underline{\Delta}^{\mathrm{u}} \mathcal{C}_{p}$, which should be called the normalization functor, works as follows: By Lemma 4.2, every lax functor $F=(F, \widehat{F}):[p] \rightsquigarrow \mathcal{C}$ uniquely determines a normal lax functor $F^{\mathrm{u}}=\left(F^{\mathrm{u}}, \hat{F}^{\mathrm{u}}\right):[p] \rightsquigarrow \mathcal{C}$, such that

$$
\begin{cases}F^{\mathrm{u}} i=F i & \text { for } 0 \leq i \leq p, \\ F_{i, j}^{\mathrm{u}}=F_{i, j} & \text { for } 0 \leq i<j \leq p, \\ \widehat{F}_{i, j, k}^{\mathrm{u}}=\widehat{F}_{i, j, k} & \text { for } 0 \leq i<j<k \leq p .\end{cases}
$$

This normal lax functor $F^{\mathrm{u}}$ is indeed the unique one such that the family of deformations in $\mathcal{C}$,

$$
\widehat{v}_{i, j}=\left\{\begin{array}{ll}
1_{F_{i, j}}: F_{i, j} \Rightarrow F_{i, j} & \text { if } i \neq j, \\
\hat{F}_{i}: 1_{F i} \Rightarrow F_{i, i} & \text { if } i=j,
\end{array} \quad 0 \leq i \leq j \leq p,\right.
$$

becomes a morphism $\widehat{v}: F^{\mathrm{u}} \Rightarrow F$ in $\underline{\Delta}^{\mathrm{u}} \mathcal{C}_{p}$. Furthermore, any morphism $\alpha: F \Rightarrow G$ in $\Delta \mathcal{C}_{p}$ uniquely determines a morphism $\alpha^{\mathrm{u}}: F^{\mathrm{u}} \Rightarrow G^{\mathrm{u}}$ in $\underline{\Delta}^{\mathrm{u}} \mathcal{C}_{p}$ such that the square

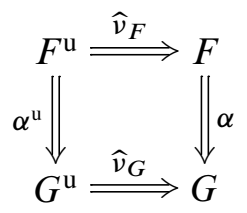

is commutative, namely,

$$
\alpha_{i, j}^{\mathrm{u}}= \begin{cases}\alpha_{i, j} & \text { if } i \neq j, \\ 1_{1_{F i}} & \text { if } i=j .\end{cases}
$$

These mappings $F \mapsto F^{\mathrm{u}}, \alpha \mapsto \alpha^{\mathrm{u}}$, describe the functor $(-)^{\mathrm{u}}: \underline{\Delta} \mathcal{C}_{p} \rightarrow \underline{\Delta}^{\mathrm{u}} \mathcal{C}_{p}$ that is right adjoint to the inclusion $\underline{\Delta}^{\mathrm{u}} \mathcal{C}_{p} \hookrightarrow \Delta \mathcal{C}_{p}$, with the identity and $\hat{v}$ being the unit and the counit of the adjunction, respectively.

In the proof of Theorem 6.4 below, our discussion uses the so-called Segal projection functors (see Segal [37, Definition 1.2]) that, in our simplicial category $\underline{\Delta}^{\mathrm{u}} \mathcal{C}$, are defined by

$$
P_{n}=\prod_{k=1}^{n} d_{0} \cdots d_{k-2} d_{k+1} \cdots d_{n}: \underline{\Delta}^{\mathrm{u}} \mathcal{C}_{n} \longrightarrow \underline{\mathrm{N}} \mathcal{C}_{n}
$$


for $n \geq 2$. That is, each

$$
P_{n}: \underline{\text { NorLaxFunc }}([n], \mathcal{C}) \rightarrow \bigsqcup_{\left(x_{0}, \ldots, x_{n}\right) \in \mathrm{Ob}^{n+1}} \mathcal{C}\left(x_{1}, x_{0}\right) \times \mathcal{C}\left(x_{2}, x_{1}\right) \times \cdots \times \mathcal{C}\left(x_{n}, x_{n-1}\right),
$$

maps a normal geometric $n$-simplex of $\mathcal{C}, F=(F, \widehat{F}):[n] \rightsquigarrow \mathcal{C}$ to the string

$$
P_{n} F=\left(F 0 \stackrel{F_{0,1}}{\longleftarrow} F 1 \stackrel{F_{1,2}}{\longleftarrow} \cdots \stackrel{F_{n-1, n}}{\longleftarrow} F n\right),
$$

and a morphism $\hat{\alpha}: F \Rightarrow F^{\prime}$ in $\underline{\Delta}^{\mathrm{u}} \mathcal{C}_{n}$ to

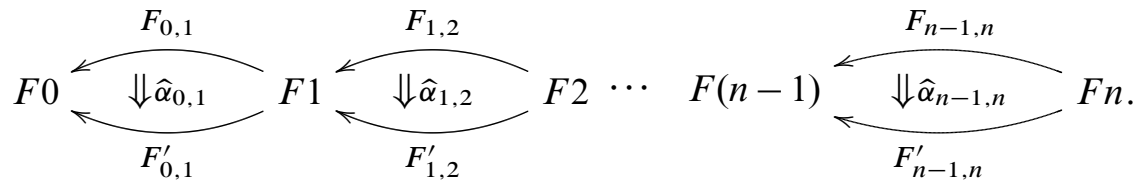

Theorem 6.4 For any bicategory $\mathcal{C}$, the pseudosimplicial embeddings $J: \underline{N} \mathcal{C} \rightsquigarrow \underline{\Delta}^{\mathrm{u}} \mathcal{C}$ and $J: \underline{N C} \rightsquigarrow \underline{S} \mathcal{C}$ induce both homotopy equivalences on the corresponding classifying spaces; thus, $\mathrm{BC} \simeq \mathrm{B} \underline{\Delta}^{\mathrm{u}} \mathcal{C}$ and $\mathrm{BC} \simeq \mathrm{B} \underline{\mathrm{S}} \mathcal{C}$.

Proof For $n=0$, 1, we have equalities

$$
\begin{aligned}
& \underline{\Delta}^{\mathrm{u}} \mathcal{C}_{0}=\underline{\mathrm{S}} \mathcal{C}_{0}=\mathrm{Ob} \mathcal{C}=\underline{\mathrm{N}} \mathcal{C}_{0} \\
& \underline{\Delta}^{\mathrm{u}} \mathcal{C}_{1}=\underline{\mathrm{S}} \mathcal{C}_{1}=\bigsqcup_{x_{0}, x_{1}} \mathcal{C}\left(x_{1}, x_{0}\right)=\underline{\mathrm{N}} \mathcal{C}_{1}
\end{aligned}
$$

by identifying a normal lax functor $F$ : [0] $\rightsquigarrow \mathcal{C}$ with the object $F 0$, and a normal lax functor $F$ : [1] $\rightsquigarrow \mathcal{C}$ with the arrow $F_{0,1}: F 1 \rightarrow F 0$, and the functors $J_{0}$ and $J_{1}$ are both identities.

For $n \geq 2$, we have the equality $P_{n} J_{n}=1$. Moreover, there is a natural transformation $\widehat{\varepsilon}: J_{n} P_{n} \Rightarrow 1$ defined as follows: If $F:[n] \rightsquigarrow \mathcal{C}$ is any normal lax functor, that is, any object of the category $\underline{\Delta}^{\mathrm{u}} \mathcal{C}_{n}$, and $J_{n} P_{n}(F)=F^{\mathrm{e}}$ as in (48), then $\widehat{\varepsilon}: F^{\mathrm{e}} \Rightarrow F$ is the morphism in $\Delta^{\mathrm{u}} \mathcal{C}_{n}$ given by the family of 2 -cells

$$
F j \sqrt{\underbrace{}_{F_{i, j}} F_{\widehat{\varepsilon}_{i, j}}^{\mathrm{e}}} F i, \quad 0 \leq i \leq j \leq n,
$$


where $\widehat{\varepsilon}_{i, i}=1_{1_{F i}}, \widehat{\varepsilon}_{i, i+1}=1_{F_{i, i+1}}$, and then inductively defined by pasting the diagrams

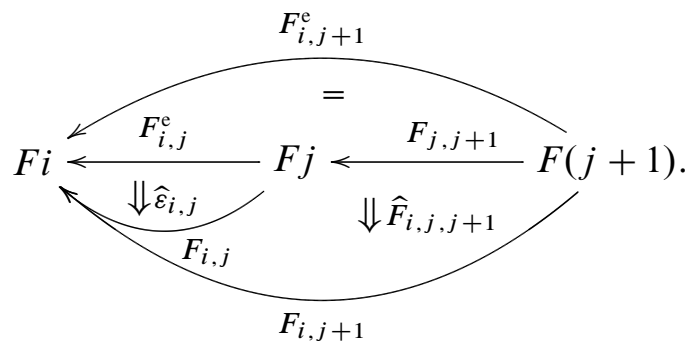

Since $P_{n} \widehat{\varepsilon}=1_{P_{n}}$ and $\widehat{\varepsilon} J_{n}=1_{J_{n}}$, it follows that $P_{n}: \underline{\Delta}^{\mathrm{u}} \mathcal{C}_{n} \rightarrow \underline{\mathrm{N}} \mathcal{C}_{n}$ is a right adjoint functor to $J_{n}$ : $\underline{\mathrm{N}}_{n} \rightarrow \underline{\Delta}^{\mathrm{u}} \mathcal{C}_{n}$, with the identity and $\varepsilon$ being the unit and the counit of the adjunction, respectively.

It follows that every $J_{n}: \underline{N}_{n} \rightarrow \underline{\Delta}^{\mathrm{u}} \mathcal{C}_{n}, n \geq 0$, induces a homotopy equivalence on classifying spaces $\mathrm{BN} \mathcal{C}_{n} \rightarrow \mathrm{B} \underline{\Delta}^{\mathrm{u}} \mathcal{C}_{n}$ whence, by Theorem 2.2 , the induced by the pseudosimplicial functor $\mathrm{B} J: \mathrm{BC}=\mathrm{B} \underline{N} \mathcal{C} \rightarrow \mathrm{B} \underline{\Delta}^{\mathrm{u}} \mathcal{C}$ so is.

Finally we restrict the above constructions to the simplicial subcategory $\underline{S} \mathcal{C} \subseteq \underline{\Delta}^{\mathrm{u}} \mathcal{C}$. Note that, when $F:[n] \rightsquigarrow \mathcal{C}$ is a normal homomorphism, that is, an object of $\underline{S} \mathcal{C}_{n}$, then $\widehat{\varepsilon}: J_{n} P_{n}(F) \Rightarrow F$ is an isomorphism. It follows that every functor $J_{n}: \underline{\mathrm{N}} \mathcal{C}_{n} \rightarrow \underline{\mathrm{S}}_{n}$ is an equivalence of categories, with $P_{n}$ : $\underline{\mathrm{S}}_{n} \rightarrow \underline{\mathrm{N}} \mathcal{C}_{n}$ being a quasi-inverse for $n \geq 2$, and therefore $J: \underline{N C} \rightsquigarrow \underline{S} \underline{C}$ is actually a pseudosimplicial equivalence. Hence, the induced map $\mathrm{B} J: \mathrm{BC} \rightarrow \mathrm{B} \underline{S} \mathcal{C}$ is a homotopy equivalence, as claimed.

Since the triangle

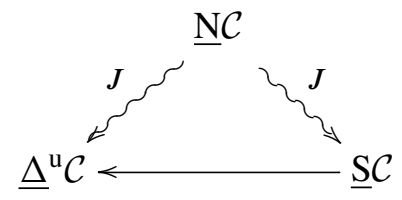

commutes, we deduce the following consequence of the above Theorem 6.4, which makes the proof for Theorem 6.1 complete:

Theorem 6.5 For any bicategory $\mathcal{C}$, the simplicial inclusion functor $\underline{S} \mathcal{C} \hookrightarrow \underline{\Delta}^{\mathrm{u}} \mathcal{C}$ induces a homotopy equivalence on classifying spaces; thus, $\mathrm{B} \underline{\mathcal{C}} \simeq \mathrm{B} \underline{\Delta}^{\mathrm{u}} \mathcal{C}$.

We should stress here that it is not true that the simplicial set of objects of Segal's nerve $\underline{\mathrm{S}} \mathcal{C}$ of a bicategory $\mathcal{C}$, that is, the simplicial set $\mathrm{SC}:[p] \mapsto \operatorname{NorHom}([p], \mathcal{C})$, represents the homotopy type of the bicategory, in contrast to what happens with the categorical geometric nerves. 
Remark 6.6 Let $\Omega^{-1} \mathcal{M}$ be the delooping bicategory of a monoidal category $\mathcal{M}=$ $(\mathcal{M}, \otimes, \mathrm{I}, \boldsymbol{a}, \boldsymbol{l}, \boldsymbol{r})$ (see Remark 3.3). The simplicial space $[n] \mapsto \mathrm{B}\left(\underline{\mathrm{S}} \Omega^{-1} \mathcal{M}_{n}\right)$ satisfies the hypothesis of Segal's Proposition 1.5 in [37], that is, the space $\mathrm{B} \underline{S} \Omega^{-1} \mathcal{M}_{0}$ is contractible (since it is a one point space) and the projection maps

$$
p_{n}=\mathrm{B} P_{n}: \mathrm{B}\left(\underline{\mathrm{S}} \Omega^{-1} \mathcal{M}_{n}\right) \rightarrow\left(\mathrm{B} \underline{\mathrm{S}} \Omega^{-1} \mathcal{M}_{1}\right)^{n}=\mathrm{B} \mathcal{M}^{n}
$$

are homotopy equivalences, where $\mathrm{B} \mathcal{M}$ is the classifying space of the underlying category $\mathcal{M}$ (since every $P_{n}: \underline{\mathrm{S}} \Omega^{-1} \mathcal{M}_{n} \rightarrow \mathcal{M}^{n}$ is an equivalence of categories, as we observed in the proof of Theorem 6.4 above). Therefore, $\Omega\left(\mathrm{B} \Omega^{-1} \mathcal{M}, *\right)$ is a group completion of $\mathrm{B} \mathcal{M}$.

Note that the same conclusion, with the same discussion as above, can be obtained by using any of the unitary categorical geometric nerves of the delooping bicategory $\Omega^{-1} \mathcal{M}$, that is, $\underline{\Delta}^{\mathrm{u}} \Omega^{-1} \mathcal{M}$ or $\bar{\nabla}_{\mathrm{u}} \Omega^{-1} \mathcal{M}$ instead of the Segal nerve for modeling $\mathrm{B} \Omega^{-1} \mathcal{M}$.

\section{The homotopy colimit theorem for bicategories}

Many properties of the classifying space construction for bicategories, $\mathcal{C} \mapsto \mathrm{BC}$, are easily established by using geometric nerves. For example:

Proposition 7.1 (i) Any lax (resp. oplax) functor between bicategories $F: \mathcal{B} \rightsquigarrow$ $\mathcal{C}$ induces a continuous map $\mathrm{B} F: \mathrm{B} \mathcal{B} \rightarrow \mathrm{BC}$, well defined up to homotopy equivalence.

(ii) If two lax (resp. oplax) functors between bicategories, $F, F^{\prime}: \mathcal{B} \rightsquigarrow \mathcal{C}$, are related by a lax or an oplax transformation, $F \Rightarrow F^{\prime}$, then the induced maps on classifying spaces, $\mathrm{B} F, \mathrm{~B} F^{\prime}: \mathrm{B} \mathcal{B} \rightarrow \mathrm{BC}$, are homotopic.

(iii) If a homomorphism of bicategories has a left or right biadjoint, then the induced map on classifying spaces is a homotopy equivalence. In particular, any biequivalence of bicategories induces a homotopy equivalence on classifying spaces.

Proof (i) The geometric nerve construction on bicategories $\mathcal{C} \mapsto \Delta \mathcal{C}$ (resp. $\mathcal{C} \mapsto \nabla \mathcal{C}$ ) is functorial on lax (resp. oplax) functors between bicategories. Therefore, Theorem 6.1 gives the result. 
(ii) Let $F, F^{\prime}: \mathcal{B} \rightsquigarrow \mathcal{C}$ be lax functors and suppose $\alpha: F \Rightarrow F^{\prime}$ is a lax transformation. There is a lax functor $H: \mathcal{B} \times[1] \rightsquigarrow \mathcal{C}$ making the diagram commutative

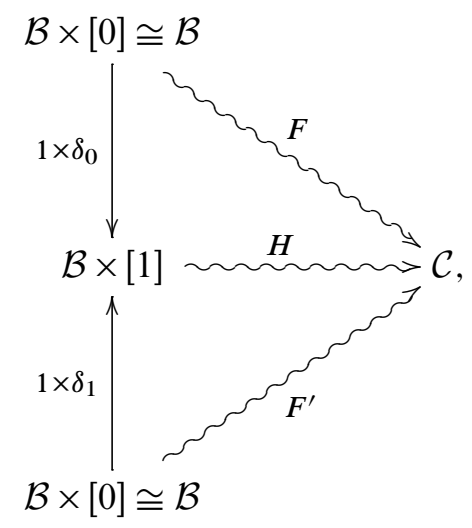

that carries a morphism in $\mathcal{B} \times[1]$ of the form $(u: x \rightarrow y, 1 \rightarrow 0):(x, 1) \rightarrow(y, 0)$ to the composite morphism in $\mathcal{C}$

$$
F x \stackrel{\alpha x}{\longrightarrow} F^{\prime} x \stackrel{F^{\prime} u}{\longrightarrow} F^{\prime} y
$$

and a deformation $\left(\phi, 1_{1 \rightarrow 0}\right):(u: x \rightarrow y, 1 \rightarrow 0) \Rightarrow(v: x \rightarrow y, 1 \rightarrow 0)$ to the composite

$$
F^{\prime} \phi \circ 1_{\alpha x}: F^{\prime} u \circ \alpha x \Rightarrow F^{\prime} v \circ \alpha x .
$$

For $u: x \rightarrow y$ and $v: y \rightarrow z$ two composable morphisms in $\mathcal{B}$, the structure constraint

$$
\hat{H}: H(y \stackrel{v}{\rightarrow} z, 1 \rightarrow 0) \circ H(x \stackrel{u}{\rightarrow} y, 1 \rightarrow 1) \Rightarrow H(x \stackrel{v \circ u}{\longrightarrow} z, 1 \rightarrow 0)
$$

is the deformation obtained by pasting the diagram

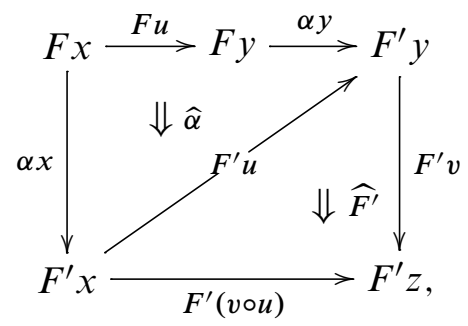

whereas the constraint

$$
\hat{H}: H(y \stackrel{v}{\rightarrow} z, 0 \rightarrow 0) \circ H(x \stackrel{u}{\rightarrow} y, 1 \rightarrow 0) \Rightarrow H(x \stackrel{v \circ u}{\rightarrow} z, 1 \rightarrow 0)
$$


is the composite deformation

$$
F^{\prime} v \circ\left(F^{\prime} v \circ \alpha x\right) \stackrel{a}{\Longrightarrow}\left(F^{\prime} v \circ F^{\prime} u\right) \circ \alpha x \stackrel{\widehat{F}^{\prime} \circ 1}{\Longrightarrow} F^{\prime}(v \circ u) \circ \alpha x .
$$

Applying geometric nerve construction to diagram (56), we get a diagram of simplicial set maps

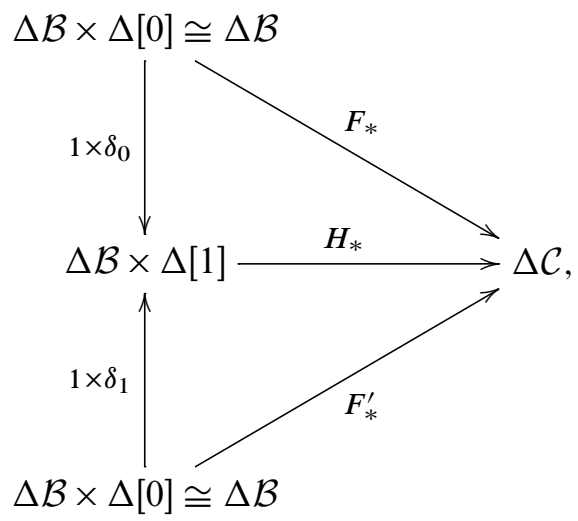

showing that the simplicial maps $F_{*}, F_{*}^{\prime}: \Delta \mathcal{B} \rightarrow \Delta \mathcal{C}$ are made homotopic by $H_{*}$, whence the result follows by Theorem 6.1.

The proof is similar for the case in which $\alpha: F \Rightarrow F^{\prime}: \mathcal{B} \rightsquigarrow \mathcal{C}$ is an oplax transformation, but with a change in the construction of the lax functor $H: \mathcal{B} \times[1] \rightsquigarrow \mathcal{C}$ that makes diagram (56) commutative: now define $H$ such that

$$
H(x \stackrel{u}{\rightarrow} y, 1 \rightarrow 0)=\alpha y \circ F u: F x \rightarrow F^{\prime} y .
$$

The statements in (iii) follow from (i) and (ii).

In this section, we mainly want to show how the use of geometric nerves and Theorem 6.1 can be applied to homotopy theory of diagrams of bicategories $\mathcal{C}: I^{\text {op }} \rightarrow$ Bicat, through a bicategorical Grothendieck construction

$$
\int_{I} \mathcal{C}
$$

which assembles all bicategories $\mathcal{C}_{i}$ as we explain next. Recall that Bicat, here, means the category of bicategories with homomorphisms.

Definition 7.2 Let $\mathcal{C}: I^{\mathrm{op}} \rightarrow$ Bicat be a functor, $(a: j \rightarrow i) \mapsto\left(a^{*}: \mathcal{C}_{i} \rightarrow \mathcal{C}_{j}\right)$, where $I$ is a small category. Then, the Grothendieck construction on $\mathcal{C}$ is the bicategory, denoted by $\int_{I} \mathcal{C}$, whose objects are pairs $(x, i)$, where $i$ is an object of $I$ and $x$ is one of the bicategory $\mathcal{C}_{i}$. A morphism $(u, a):(y, j) \rightarrow(x, i)$ in $\int_{I} \mathcal{C}$ is a pair 
of morphisms where $a: j \rightarrow i$ in $I$ and $u: y \rightarrow a^{*} x$ in $\mathcal{C}_{j}$. Given two morphisms $(u, a),\left(u^{\prime}, a^{\prime}\right):(y, j) \rightarrow(x, i)$, the existence of a deformation $(u, a) \Rightarrow\left(u^{\prime}, a^{\prime}\right)$ requires that $a=a^{\prime}$, and then, such a deformation

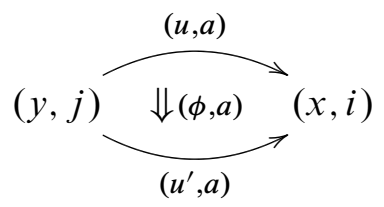

consists of a deformation

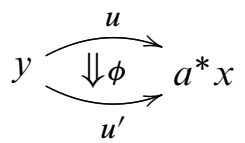

in $\mathcal{C}_{j}$. Thus, $\mathrm{Ob} \int_{I} \mathcal{C}=\bigsqcup_{i \in \mathrm{Ob} I} \mathrm{Ob} \mathcal{C}_{i}$, and the hom-categories of $\int_{I} \mathcal{C}$ are

$$
\int_{I} \mathcal{C}((y, j),(x, i))=\bigsqcup_{j \rightarrow i} \mathcal{C}_{j}\left(y, a^{*} x\right)
$$

where the disjoint union is over all arrows $a: j \rightarrow i$ in $I$.

For each triplet of objects $(z, k),(y, j)$ and $(x, i)$ of $\int_{I} \mathcal{C}$, the horizontal composition functor

$$
\bigsqcup_{j \rightarrow i} \mathcal{C}_{j}\left(y, a^{*} x\right) \times \bigsqcup_{\substack{b \\ \rightarrow}} \mathcal{C}_{k}\left(z, b^{*} y\right) \stackrel{\circ}{\longrightarrow} \bigsqcup_{k \stackrel{c}{\rightarrow} i} \mathcal{C}_{k}\left(z, c^{*} i\right)
$$

maps the component at two morphisms $a: j \rightarrow i$ and $b: k \rightarrow j$ of $I$ into the component at the composite $a b: k \rightarrow i$ via the composition

$$
\mathcal{C}_{j}\left(y, a^{*} x\right) \times \mathcal{C}_{k}\left(z, b^{*} y\right) \stackrel{b^{*} \times 1}{\longrightarrow} \mathcal{C}_{k}\left(b^{*} y, b^{*} a^{*} x\right) \times \mathcal{C}_{k}\left(z, b^{*} y\right) \stackrel{\circ}{\longrightarrow} \mathcal{C}_{k}\left(z, b^{*} a^{*} x\right),
$$

that is,

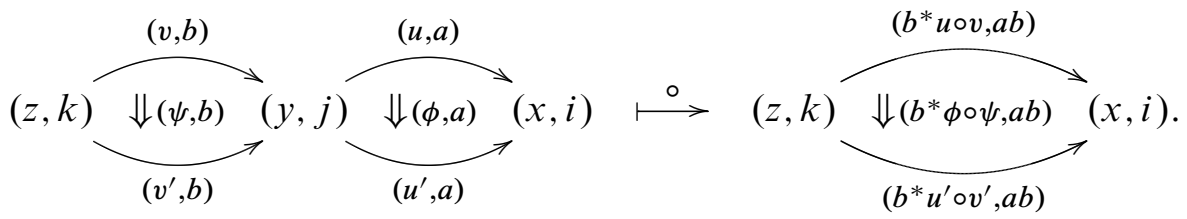

Given any three composable morphisms

$$
(t, \ell) \stackrel{(w, c)}{\longrightarrow}(z, k) \stackrel{(v, b)}{\longrightarrow}(y, j) \stackrel{(u, a)}{\longrightarrow}(x, i)
$$


in $\int_{I} \mathcal{C}$, the structure associativity isomorphism

$$
(u, a) \circ((v, b) \circ(w, c)) \cong((u, a) \circ(v, b)) \circ(w, c)
$$

is provided by pasting, in the bicategory $\mathcal{C}_{\ell}$, the diagram

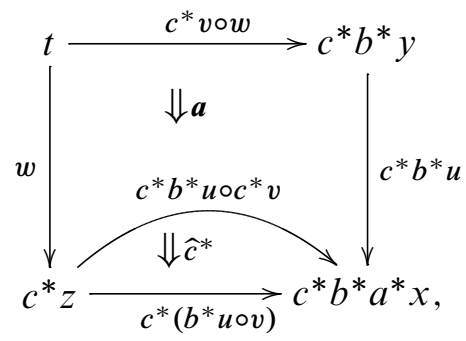

where $\boldsymbol{a}$ is the associativity isomorphism in $\mathcal{C}_{\ell}$ and $\hat{c}^{*}$ is the structure isomorphism of the homomorphism $c^{*}: \mathcal{C}_{k} \rightsquigarrow \mathcal{C}_{\ell}$.

The identity morphism for each object $(x, i)$ in $\int_{I} \mathcal{C}$ is

$$
\left(1_{x}, 1_{i}\right):(x, i) \rightarrow(x, i),
$$

and, for each morphism $(u, a):(y, j) \rightarrow(x, i)$, the left unit constraint

$$
\left(1_{x}, 1_{i}\right) \circ(u, a)=\left(a^{*} 1_{x} \circ u, a\right) \cong(u, a)
$$

is provided by the left unit constraint of $\mathcal{C}_{j}$ and the unit structure constraint of the homomorphism $a^{*}: \mathcal{C}_{i} \rightsquigarrow \mathcal{C}_{j}$ by pasting

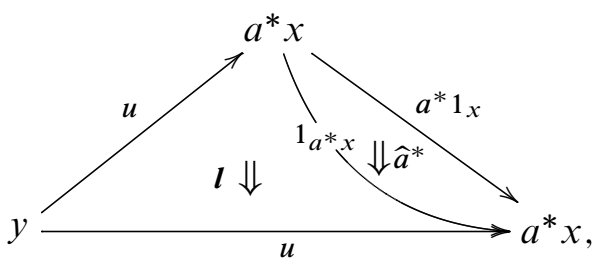

whereas the right unit constraint

$$
(u, a) \circ\left(1_{y}, 1_{j}\right)=\left(u \circ 1_{y}, a\right) \cong(u, a)
$$

is directly given by the right unit constraint $r: u \circ 1_{y} \Rightarrow u$ of $\mathcal{C}_{j}$ at $u$.

We want to remark that the above Grothendieck construction $\int_{I} \mathcal{C}$ is a particular case of that given by Baković in [2].

The well-known Homotopy Colimit Theorem by Thomason, that is, Theorem 2.1, admits the following generalization to diagrams of bicategories $\mathcal{C}: I^{\mathrm{op}} \rightarrow$ Bicat in Theorem 
7.3, where it is established how the classifying space of the bicategory Grothendieck construction $\mathrm{B} \int_{I} \mathcal{C}$ can be thought of as a homotopy colimit of the spaces $\mathrm{BC} \mathcal{C}_{i}$ that arise from the initial input data $i \mapsto \mathcal{C}_{i}$ given by the functor $\mathcal{C}$.

Theorem 7.3 Suppose a category $I$ is given. For every functor $\mathcal{C}: I^{\mathrm{op}} \rightarrow$ Bicat there exists a natural weak homotopy equivalence of simplicial sets

$$
\eta: \operatorname{hocolim}_{I} \Delta \mathcal{C} \longrightarrow \Delta \int_{I} \mathcal{C}
$$

where $\Delta \mathcal{C}: I^{\mathrm{op}} \rightarrow$ Simpl.Set is the diagram of simplicial sets obtained by composing $\mathcal{C}$ with the geometric nerve functor $\Delta:$ Bicat $\rightarrow$ Simpl.Set.

Proof To show an explicit description of the weak equivalence $\eta$ in the theorem, we shall first explicitly describe the simplicial sets hocolim $I \Delta \mathcal{C}$ and $\Delta \int_{I} \mathcal{C}$.

On one hand, the simplicial set hocolim $I \Delta \mathcal{C}$ is the diagonal of the bisimplicial set

$$
S=\bigsqcup_{G \in \Delta I} \Delta \mathcal{C}_{G 0}=\bigsqcup_{G:[q] \rightarrow I} \operatorname{LaxFunc}\left([p], \mathcal{C}_{G 0}\right),
$$

whose $(p, q)$-simplices are pairs

$$
(F, G)
$$

consisting of a functor $G:[q] \rightarrow I$ and a lax functor $F:[p] \rightsquigarrow \mathcal{C}_{G 0}$. If $\alpha:\left[p^{\prime}\right] \rightarrow[p]$ and $\beta:\left[q^{\prime}\right] \rightarrow[q]$ are maps in the simplicial category, then the respective horizontal and vertical induced maps $\alpha^{*_{h}}: S_{p, q} \rightarrow S_{p^{\prime}, q}$ and $\beta^{*_{v}}: S_{p, q} \rightarrow S_{p, q^{\prime}}$ are defined by

$$
\left\{\begin{array}{l}
\alpha^{*_{h}}(F, G)=(F \alpha, G), \\
\beta^{*_{v}}(F, \sigma)=\left(G_{0, \beta 0}^{*} F, G \beta\right),
\end{array}\right.
$$

where $G_{0, \beta 0}^{*} F:[p] \rightsquigarrow \mathcal{C}_{G \beta 0}$ is the lax functor obtained by the composition of $F$ with the homomorphism of bicategories $G_{0, \beta 0}^{*}: \mathcal{C}_{G 0} \rightsquigarrow \mathcal{C}_{G \beta 0}$ attached in diagram $\mathcal{C}: I^{\text {op }} \rightarrow$ Bicat at the morphism $G_{0, \beta 0}: G \beta 0 \rightarrow G 0$ of $I$. In particular, the horizontal face maps are given by

$$
d_{i}^{h}(F, G)=\left(F d^{i}, G\right), \quad \text { for } 0 \leq i \leq p,
$$

and the vertical ones by

while

$$
\begin{aligned}
& d_{j}^{v}(F, G)=\left(F, G d^{j}\right), \quad \text { for } 1 \leq j \leq q, \\
& d_{0}^{v}(F, G)=\left(G_{0,1}^{*} F, G d^{0}\right) .
\end{aligned}
$$


On the other hand, a $p$-simplex of $\Delta \int_{I} \mathcal{C}$ is a lax functor $[p] \rightsquigarrow \int_{I} \mathcal{C}$, which can be described as a pair

$$
\left(F^{\prime}, G\right)
$$

where $G:[p] \rightarrow I$ is a functor, that is, a $p$-simplex of $\Delta I$, and $F^{\prime}:[p] \rightsquigarrow \mathcal{C}$ is a $G$-crossed lax functor (cf [12, Section 4.1]), that is, a family

$$
F^{\prime}=\left\{F^{\prime} i, F_{i, j}^{\prime}, \hat{F}_{i, j, k}^{\prime}, \hat{F}_{i}^{\prime}\right\}_{0 \leq i \leq j \leq k \leq p}
$$

in which each $F^{\prime} i$ is an object of the bicategory $\mathcal{C}_{G i}$, each $F_{i, j}^{\prime}: F^{\prime} j \rightarrow G_{i, j}^{*} F^{\prime} i$ is a morphism in $\mathcal{C}_{G j}$, each $\hat{F}_{i, j, k}^{\prime}: G_{j, k}^{*} F_{i, j}^{\prime} \circ F_{j, k}^{\prime} \Rightarrow F_{i, k}^{\prime}$ is a deformation in $\mathcal{C}_{G k}$

$$
G_{j, k}^{*} G_{i, j}^{*} F^{\prime} i=G_{i, k}^{*} F^{\prime} i<F_{i, k}^{\prime} F_{\hat{F}_{i, j, k}^{\prime}}^{G_{j, k}^{*} F_{i, j}^{\prime} F^{*} F^{\prime} j} F^{\prime} k,
$$

and each $\hat{F}_{i}^{\prime}: 1_{F^{\prime} i} \Rightarrow F_{i, i}^{\prime}$ is a deformation in $\mathcal{C}_{G i}$. These data are required to satisfy that, for $0 \leq i \leq j \leq k \leq \ell \leq p$, the diagram of deformations

$$
\begin{aligned}
& G_{j, \ell}^{*} F_{i, j}^{\prime} \circ\left(G_{k, \ell}^{*} F_{j, k}^{\prime} \circ F_{k, \ell}^{\prime}\right) \Longrightarrow\left(G_{j, \ell}^{*} F_{i, j}^{\prime} \circ G_{k, \ell}^{*} F_{j, k}^{\prime}\right) \circ F_{k, \ell}^{\prime} \\
& 1 \circ \widehat{F}_{j, k, \ell}^{\prime}\|\quad\| \widehat{G}_{k, \ell}^{*} \\
& G_{j, \ell}^{*} F_{i, j}^{\prime} \circ F_{j, \ell}^{\prime} \quad G_{k, \ell}^{*}\left(G_{j, k}^{*} F_{i, j}^{\prime} \circ F_{j, k}^{\prime}\right) \circ F_{k, \ell}^{\prime} \\
& \widehat{F}_{i, j, \ell}^{\prime} \Downarrow \quad \| G_{k, \ell}^{*} \widehat{F}_{i, j, k}^{\prime} \circ 1 \\
& F_{i, \ell}^{\prime} \Longleftarrow \widehat{F}_{i, k, \ell}^{\prime} G_{k, \ell}^{*} F_{i, k}^{\prime} \circ F_{k, \ell}^{\prime}
\end{aligned}
$$

commutes in the bicategory $\mathcal{C}_{G \ell}$, and, for any $0 \leq i \leq j \leq p$, both diagrams below commute in $\mathcal{C}_{G j}$.

$$
\begin{array}{r}
G_{i, j}^{*} F_{i, i}^{\prime} \circ F_{i, j}^{\prime} \stackrel{G_{i, j}^{*} \hat{F}_{i}^{\prime} \circ 1}{\Longleftarrow} G_{i, j}^{*} 1_{F^{\prime} i} \circ F_{i, j}^{\prime} \\
\hat{F}_{i, i, j}^{\prime} \Downarrow \\
F_{\widehat{G}_{i, j}^{*} \circ 1} \longleftarrow \quad \boldsymbol{r} 1_{G_{i, j}^{*} F^{\prime} i} \circ F_{i, j}^{\prime}
\end{array}
$$

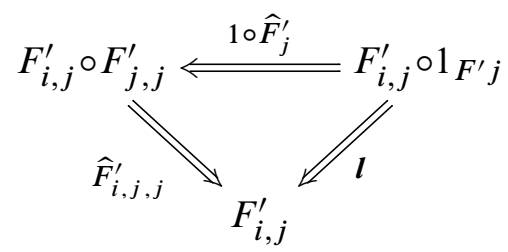

If $\alpha:\left[p^{\prime}\right] \rightarrow[p]$ is a map in the simplicial category, then the induced map

$$
\alpha^{*}:\left(\Delta \int_{I} \mathcal{C}\right)_{p} \rightarrow\left(\Delta \int_{I} \mathcal{C}\right)_{p^{\prime}}
$$


associates to a $p$-simplex $\left(F^{\prime}, G\right)$, as above, the $p^{\prime}$-simplex

$$
\alpha^{*}\left(F^{\prime}, G\right)=\left(F^{\prime} \alpha, G \alpha\right)
$$

in which $F^{\prime} \alpha=\left\{F^{\prime} \alpha i, F_{\alpha i, \alpha j}^{\prime}, \hat{F}_{\alpha_{i}, \alpha_{j}, \alpha_{k}}^{\prime}, \hat{F}_{\alpha i}^{\prime}\right\}_{0 \leq i \leq j \leq k \leq p^{\prime}}$.

The simplicial map (57)

$$
\eta: \operatorname{hocolim}_{I} \Delta \mathcal{C} \longrightarrow \Delta \int_{I} \mathcal{C}
$$

is then given on a $p$-simplex $(F, G)$ of $\operatorname{hocolim}_{I} \Delta \mathcal{C}$ by

$$
\eta(F, G)=\left(F^{\prime}, G\right)
$$

where $F^{\prime}:[p] \rightsquigarrow \mathcal{C}$ is the $G$-crossed lax functor as in (59), defined by the objects $F^{\prime} i=G_{0, i}^{*} F i$, the morphisms $F_{i, j}^{\prime}=G_{0, j}^{*} F_{i, j}: F^{\prime} j \rightarrow G_{i, j}^{*} F^{\prime} i$ and the deformations $\hat{F}_{i, j, k}^{\prime}: G_{j, k}^{*} F_{i, j}^{\prime} \circ F_{j, k}^{\prime} \Rightarrow F_{i, k}^{\prime}$ and $\widehat{F}_{i}^{\prime}: 1_{F^{\prime} i} \Rightarrow F_{i, i}^{\prime}$, which are, respectively, the composites

$$
\begin{aligned}
& \hat{F}_{i, j, k}^{\prime}: G_{0, k}^{*} F_{i, j} \circ G_{0, k}^{*} F_{j, k} \stackrel{\widehat{G}_{0, k}^{*}}{\longrightarrow} G_{0, k}^{*}\left(F_{i, j} \circ F_{j, k}\right) \stackrel{G_{0, k}^{*} \widehat{F}_{i, j, k}}{\longrightarrow} G_{0, k}^{*} F_{i, k}, \\
& \hat{F}_{i}^{\prime}: 1_{G_{0, i}^{*} F i} \stackrel{\widehat{G}_{0, i}^{*}}{\longrightarrow} G_{0, i}^{*} 1_{F i} \stackrel{G_{0, i}^{*} \hat{F}_{i}}{\longrightarrow} G_{0, i}^{*} F_{i, i} .
\end{aligned}
$$

To prove that this map $\eta$ is a weak equivalence, our strategy now is to apply the weak homotopy equivalences (2) on the bisimplicial set $S$, defined in (58). Since $\operatorname{diag} S=$ $\operatorname{hocolim}_{I} \Delta \mathcal{C}$, we have a weak homotopy equivalence $\Phi: \operatorname{hocolim}_{I} \Delta \mathcal{C} \rightarrow \bar{W} S$, and the proof will be complete once we show a simplicial isomorphism

$$
\Psi: \bar{W} S \cong \Delta \int_{I} \mathcal{C}
$$

making the diagram of simplicial sets commutative:

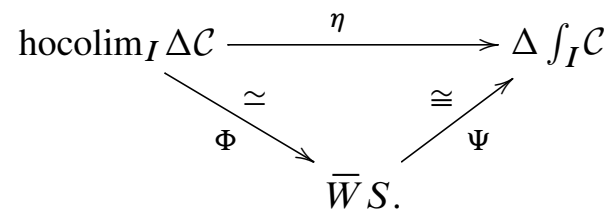

For, note that a $p$-simplex of $\bar{W} S$, say $\chi$, can be described as a list of pairs

$$
\chi=\left(\left(F^{(0}, G^{(p)}\right), \ldots,\left(F^{(m)}, G^{(p-m}\right), \ldots,\left(F^{(p}, G^{(0)}\right)\right),
$$


in which each $G^{(p-m}:[p-m] \rightarrow I$ is a functor and each $F^{(m}:[m] \rightsquigarrow \mathcal{C}_{G^{(p-m} 0}$ is a lax functor, such that the equalities

$$
G^{(p-m} d^{0}=G^{(p-m-1}, \quad F^{(m+1} d^{m+1}=G_{0,1}^{(p-m *} F^{(m)},
$$

hold for all $0 \leq m<p$. Writing $G^{(p}:[p] \rightarrow I$ simply as $G:[p] \rightarrow I$, an iterated use of the above equalities proves that

$$
G^{(p-m}=G d^{0} \stackrel{(m}{*} d^{0}:[p-m] \rightarrow I,
$$

for $0 \leq m \leq p$, and

$$
F^{(m+1} d^{m+1} \cdots d^{k+1}=G_{k, m+1}^{*} F^{(k}:[k] \rightsquigarrow \mathcal{C}_{G(m+1)},
$$

for $0 \leq k \leq m<p$. These latter equations mean that

$$
\begin{cases}F^{(j} i=G_{i, j}^{*} F^{(i} i & \text { for } i \leq j, \\ F_{i, j}^{(k}=G_{j, k}^{*} F_{i, j}^{(j} & \text { for } i \leq j \leq k, \\ \hat{F}_{i, j, k}^{(\ell}=G_{k, \ell}^{*} \widehat{F}_{i, j, k}^{(k} & \text { for } i \leq j \leq k \leq \ell, \\ \hat{F}_{i}^{(j}=G_{i, j}^{*} \widehat{F}_{i}^{(i} & \text { for } i \leq j,\end{cases}
$$

whence we see how the $p$-simplex $\chi$ of $\bar{W} S$ is uniquely determined by $G:[p] \rightarrow I$, the objects $F^{(i} i$ of $\mathcal{C}_{G i}$, the morphisms $F_{i, j}^{(j}: F^{(j} j \rightarrow F^{(j} i=G_{i, j}^{*} F^{(i} i$ of $\mathcal{C}_{G j}$, and the deformations $\widehat{F}_{i, j, k}^{(k}: F_{i, j}^{(k} \circ F_{j, k}^{(k}=G_{j, k}^{*} F_{i, j}^{*} \circ F_{j, k}^{(k} \Rightarrow F_{i, k}^{(k}$ in $\mathcal{C}_{G k}$, and $\hat{F}_{i}^{(i}: 1_{F^{(i} i} \Rightarrow F_{i, i}^{(i}$ in $\mathcal{C}_{G i}$, all for $0 \leq i \leq j \leq k \leq p$. At this point, we observe that there is a normal $G$-crossed lax functor $F^{\prime}=\left\{F^{\prime} i, F_{i, j}^{\prime}, \hat{F}_{i, j, k}^{\prime}, \hat{F}_{i}^{\prime}\right\}:[p] \rightsquigarrow \mathcal{C}$, as in (59), defined just by putting

$$
F^{\prime} i=F^{(i} i, \quad F_{i, j}^{\prime}=F_{i, j}^{(j}, \quad \hat{F}_{i, j, k}^{\prime}=F_{i, j, k}^{(k} \quad \text { and } \quad \hat{F}_{i}^{\prime}=F_{i}^{i}
$$

(the commutativity of diagrams (60) and (61) follows from $F^{(\ell}$ and $F^{(j}$ being lax functors). Thus, the $p$-simplex $\chi \in \bar{W} S$ defines the $p$-simplex $\left(F^{\prime}, G\right)$ of $\Delta \int_{I} \mathcal{C}$, which itself uniquely determines $\chi$.

In this way, we get an injective simplicial map

$$
\begin{gathered}
\Psi: \bar{W} S \rightarrow \Delta \int_{I} \mathcal{C} \\
\left(\left(F^{(0}, G^{(p)}\right), \ldots,\left(F^{(p}, G^{(0}\right)\right) \mapsto\left(F^{\prime}, G\right)=\left(\left\{F^{(i} i, F_{i, j}^{(j}, \hat{F}_{i, j, k}^{(k)}, \hat{F}_{i}^{(i}\right\}, G^{(p)}\right)
\end{gathered}
$$

which is also surjective, that is, actually an isomorphism, as we can see by retracing our steps: 
To any pair $\left(F^{\prime}, G\right)$ describing a $p$-simplex of $\Delta \int_{I} \mathcal{C}$, that is, with $G:[p] \rightarrow I$ a functor and $F^{\prime}=\left\{F^{\prime} i, F_{i, j}^{\prime}, \hat{F}_{i, j, k}^{\prime}, \hat{F}_{i}^{\prime}\right\}:[p] \rightsquigarrow \mathcal{C}$ a $G$-crossed lax functor, we associate the $p$-simplex

$$
\chi=\left(\left(F^{(m}, G^{(p-m)}\right)\right)
$$

of $\bar{W} S$, where, for each $0 \leq m \leq p, G^{(p-m}:[p-m] \rightarrow I$ is the composite

$$
[p-m] \stackrel{\left(d^{0}\right)^{m}}{\rightarrow}[p] \stackrel{G}{\rightarrow} I
$$

and the lax functor $F^{(m}:[m] \rightsquigarrow \mathcal{C}_{G^{(p-m} 0}$ is defined by the objects $F_{i=}^{(m}{ }_{i, m}^{*} F^{\prime} i$, the morphisms

$$
F_{i, j}^{(m}=G_{j, m}^{*} F_{i, j}^{\prime}: F_{j}^{(m)} \rightarrow F^{(m} i
$$

and the deformations

$$
\widehat{F}_{i, j, k}^{(m}: F_{i, j}^{(m} \circ F_{j, k}^{(m} \Rightarrow F_{i, k}^{(m)} \quad \text { and } \quad F_{i}^{(m}: 1_{F^{\left(m_{i}\right.}} \Rightarrow F_{i, i}^{(m}
$$

which are respectively given as the compositions

$$
\begin{aligned}
& \widehat{F}_{i, j, k}^{(m}: G_{j, m}^{*} F_{i, j}^{\prime} \circ G_{k, m}^{*} F_{j, k}^{\prime} \stackrel{\widehat{G}_{k, m}^{*}}{\longrightarrow} G_{k, m}^{*}\left(G_{j, k}^{*} F_{i, j}^{\prime} \circ F_{j, k}^{\prime}\right) \stackrel{G_{k, m}^{*} \widehat{F}_{i, j, k}^{\prime}}{=} G_{k, m}^{*} F_{i, k}^{\prime}, \\
& \hat{F}_{i}^{(m}: 1_{G_{i, m}^{*} F^{\prime} i} \stackrel{\widehat{G}_{i, m}^{*}}{\longrightarrow} G_{i, m}^{*} 1_{F^{\prime} i} \stackrel{G_{i, m}^{*} \widehat{F}_{i}^{\prime}}{\longrightarrow} G_{i, m}^{*} F_{i, i}^{\prime} .
\end{aligned}
$$

One easily checks that $\Psi(\chi)=\left(F^{\prime}, G\right)$, whence we conclude that the simplicial map $\Psi$ is an isomorphism as claimed.

Finally, since the map $\eta$ in the theorem occurs in the commutative diagram (62), that is, $\eta=\Psi \Phi$, where $\Psi$ is an isomorphism and $\Phi$ a weak homotopy equivalence, the proof is complete.

For any functor $\mathcal{C}: I^{\mathrm{op}} \rightarrow$ Bicat, the bicategory $\int_{I} \mathcal{C}$ assembles all bicategories $\mathcal{C}_{i}$ in the following precise sense: There is a projection 2 -functor

$$
\pi: \int_{I} \mathcal{C} \rightarrow I
$$

given by

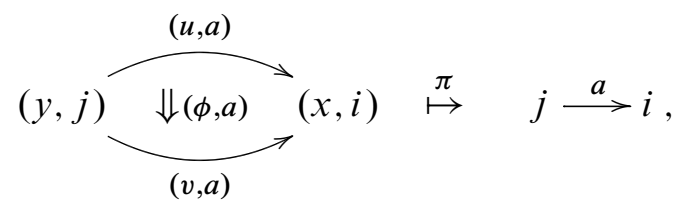


and, for each object $j$ of $I$, there is a pullback square of bicategories

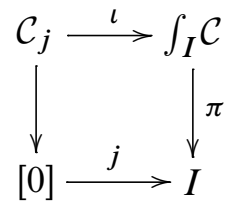

where $\iota: \mathcal{C}_{j} \rightarrow \int_{I} \mathcal{C}$ is the embedding homomorphism defined by

$$
y \frac{u}{\frac{\Downarrow_{\phi}}{v} x} \stackrel{\iota}{\mapsto}(y, j) \underbrace{\frac{\left(u, 1_{j}\right)}{\Downarrow\left(\phi, 1_{j}\right)} \rightarrow}_{\left(v, 1_{j}\right)}(x, j) .
$$

Thus, $\mathcal{C}_{j} \cong \pi^{-1}(j)$, the fibre bicategory of $\pi$ at $j$.

After Quillen's Lemma [35, page 90] (see also Goerss and Jardine [19, Section IV, Lemma 5.7]), the following result is a consequence of our Theorem 7.3:

Theorem 7.4 Suppose that $\mathcal{C}: I^{\mathrm{op}} \rightarrow$ Bicat is a diagram of bicategories such that the induced map $\mathrm{B} a^{*}: \mathrm{BC}_{i} \rightarrow \mathrm{BC}_{j}$, for each morphism $a: j \rightarrow i$ in $I$, is a homotopy equivalence. Then, for every object $j$ of $I$, the square induced by (63) on classifying spaces

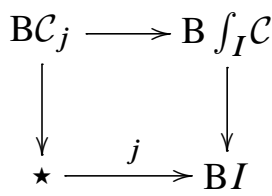

is homotopy cartesian. Therefore, for each object $y \in \mathcal{C}_{j}$ there is an induced long exact sequence on homotopy groups, relative to the base points $y$ of $\mathrm{B} \mathcal{C}_{j},(y, j)$ of $\mathrm{B} \int_{I} \mathcal{C}$, and $j$ of $\mathrm{B} I$,

$$
\cdots \rightarrow \pi_{n+1} \mathrm{~B} I \rightarrow \pi_{n} \mathrm{BC}{ }_{j} \rightarrow \pi_{n} \mathrm{~B} \int_{I} \mathcal{C} \rightarrow \pi_{n} \mathrm{~B} I \rightarrow \cdots .
$$

Remark 7.5 Let $\mathcal{M}: I^{\mathrm{op}} \rightarrow$ MonCat, $(a: j \rightarrow i) \mapsto\left(a^{*}: \mathcal{M}_{i} \rightarrow \mathcal{M}_{j}\right)$, be a diagram of monoidal categories. It follows from Theorem 6.1 and Theorem 7.3 that the homotopy type of $\mathcal{M}$ is modeled by the bicategory

$$
\int_{I} \Omega^{-1} \mathcal{M}
$$

where $\Omega^{-1}$ : MonCat $\rightarrow$ Bicat is the delooping embedding (see Remark 3.3 and Remark 5.1). 
Notice that $\int_{I} \Omega^{-1} \mathcal{M}$ is a genuine bicategory: It has the same objects $i$ as the category $I$, its hom-categories are

$$
\int_{I} \Omega^{-1} \mathcal{M}(j, i)=\bigsqcup_{j \rightarrow i} \mathcal{M}_{j}=\mathcal{M}_{j} \times I(j, i)
$$

where $\mathcal{M}_{j}$ is the underlying category of the monoidal category equally denoted, and its horizontal compositions are given by

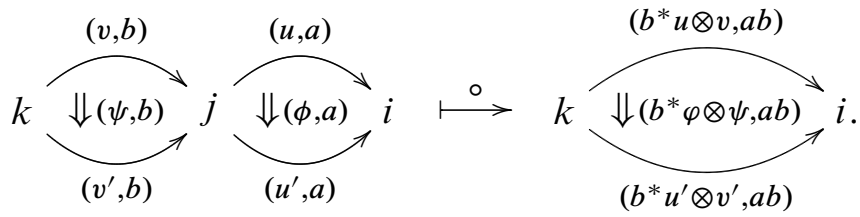

Hence, the reader interested in the study of classifying spaces of monoidal categories can find in the above fact a good reason to also be interested in the study of classifying spaces of bicategories.

Recall that a notion of nerve $\operatorname{Ner}_{I} \mathcal{M}$, for a diagram of monoidal categories $\mathcal{M}: I^{\mathrm{op}} \rightarrow$ MonCat, was defined in $[12,(66)]$ as follows: A "2-cocycle" of $I$ with coefficients in $\mathcal{M}$ is a system of data $(Y, f)$ consisting of:

- For each arrow $a: j \rightarrow i$ in $I$, an object $Y_{a} \in \mathcal{M}_{j}$.

- For each pair of composable arrows in $I, b: k \rightarrow j$ and $a: j \rightarrow i$, a morphism in $\mathcal{M}_{k}$

$$
b^{*} Y_{a} \otimes Y_{b} \stackrel{f_{a, b}}{\longrightarrow} Y_{a b},
$$

such that $Y_{1_{j}}=\mathrm{I}$ (the unit object of $\mathcal{M}_{j}$ ), the morphisms $f_{1, a}: a^{*} \mathrm{I} \otimes Y_{a} \rightarrow Y_{a}$ and $f_{a, 1}: Y_{a} \otimes \mathrm{I} \rightarrow Y_{a}$ are the canonical isomorphisms given by the unit constrains of the monoidal category $\mathcal{M}_{j}$ and the monoidal functor $a^{*}$, and for any three composable triplet, $c: \ell \rightarrow k, b: k \rightarrow j$ and $a: j \rightarrow i$, of morphisms in $I$, the diagram in $\mathcal{M}_{\ell}$

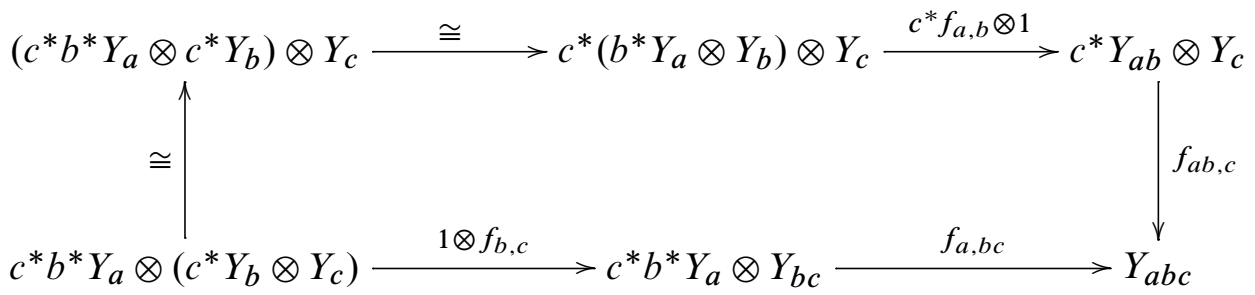


(where the unnamed isomorphisms are canonical) is commutative. Then, $\operatorname{Ner}_{I} \mathcal{M}$, the nerve of the diagram, is defined as the simplicial set

$$
\operatorname{Ner}_{I} \mathcal{M}:[n] \mapsto \bigsqcup_{G:[n] \rightarrow I} Z^{2}([n], \mathcal{M} G),
$$

where $G:[n] \rightarrow I$ is any functor and $Z^{2}([n], \mathcal{M} G)$ is the set of $2-$ cocycles of $[n]$ in the composition of the functors $G:[n] \rightarrow I$ and $\mathcal{M}: I \rightarrow$ MonCat.

It is a fact that this simplicial set (64) actually represents the homotopy type of the diagram of monoidal categories $\mathcal{M}$ since a straightforward comparison shows the existence of a natural isomorphism of simplicial sets

$$
\operatorname{Ner}_{I} \mathcal{M} \cong \Delta^{\mathrm{u}} \int_{I} \Omega^{-1} \mathcal{M}
$$

between $\operatorname{Ner}_{I} \mathcal{M}$ and the normal geometric nerve (31) of the bicategory $\int_{I} \Omega^{-1} \mathcal{M}$.

\section{References}

[1] M Artin, B Mazur, On the van Kampen theorem, Topology 5 (1966) 179-189 MR0192495

[2] I Baković, Grothendieck construction for bicategories, Preprint Available at http:// www.irb.hr/korisnici/ibakovic/sgc.pdf

[3] J Bénabou, Introduction to bicategories, from: "Reports of the Midwest Category Seminar", Springer, Berlin (1967) 1-77 MR0220789

[4] A K Bousfield, D M Kan, Homotopy limits, completions and localizations, Lecture Notes in Math. 304, Springer, Berlin (1972) MR0365573

[5] L Breen, Théorie de Schreier supérieure, Ann. Sci. École Norm. Sup. (4) 25 (1992) 465-514 MR1191733

[6] M Bullejos, A M Cegarra, On the geometry of 2-categories and their classifying spaces, $K$-Theory 29 (2003) 211-229 MR2028502

[7] M Bullejos, A M Cegarra, Classifying spaces for monoidal categories through geometric nerves, Canad. Math. Bull. 47 (2004) 321-331 MR2072592

[8] P Carrasco, A M Cegarra, (Braided) tensor structures on homotopy groupoids and nerves of (braided) categorical groups, Comm. Algebra 24 (1996) 3995-4058 MR1414569

[9] P Carrasco, A M Cegarra, Schreier theory for central extensions of categorical groups, Comm. Algebra 24 (1996) 4059-4112 MR1414570 
[10] A M Cegarra, M Bullejos, A R Garzón, Higher-dimensional obstruction theory in algebraic categories, J. Pure Appl. Algebra 49 (1987) 43-102 MR920515

[11] A M Cegarra, A R Garzón, Homotopy classification of categorical torsors, Appl. Categ. Structures 9 (2001) 465-496 MR1865612

[12] A M Cegarra, E Khmaladze, Homotopy classification of graded Picard categories, Adv. Math. 213 (2007) 644-686 MR2332605

[13] A M Cegarra, J Remedios, The relationship between the diagonal and the bar constructions on a bisimplicial set, Topology Appl. 153 (2005) 21-51 MR2172033

[14] A M Cegarra, J Remedios, The behaviour of the $\bar{W}$-construction on the homotopy theory of bisimplicial sets, Manuscripta Math. 124 (2007) 427-457 MR2357792

[15] J W Duskin, Simplicial matrices and the nerves of weak $n$-categories. I. Nerves of bicategories, Theory Appl. Categ. 9 (2001) 198-308 MR1897816 CT2000 Conference (Como)

[16] Z Fiedorowicz, Classifying spaces of topological monoids and categories, Amer. J. Math. 106 (1984) 301-350 MR737777

[17] R Garner, N Gurski, The low-dimensional structures that tricategories form, to appear in Mathematical Proc. Camb. Phil. Soc. arXiv:0711.1761

[18] P G Glenn, Realization of cohomology classes in arbitrary exact categories, J. Pure Appl. Algebra 25 (1982) 33-105 MR660389

[19] P G Goerss, J F Jardine, Simplicial homotopy theory, Progress in Math. 174, Birkhäuser Verlag, Basel (1999) MR1711612

[20] R Gordon, A J Power, R Street, Coherence for tricategories, Mem. Amer. Math. Soc. 117 (1995) vi+81 MR1261589

[21] A Grothendieck, Catégories fibrées et déscente, from: "Revêtements étales et groupe fondamental", Lecture Notes in Math. 224, Springer, Berlin (1971) 145-194 MR0354651 Séminaire de Géométrie Algébrique du Bois Marie 1960-1961 (SGA 1)

[22] N Gurski, An algebraic theory of tricategory, PhD thesis, University of Chicago (2007)

[23] N Gurski, Nerves of bicategories as stratified simplicial sets, J. Pure Appl. Algebra 213 (2009) 927-946 MR2498786

[24] V A Hinich, V V Schechtman, Geometry of a category of complexes and algebraic K-theory, Duke Math. J. 52 (1985) 399-430 MR792180

[25] J F Jardine, Supercoherence, J. Pure Appl. Algebra 75 (1991) 103-194 MR1138365

[26] A Joyal, R Street, Braided tensor categories, Adv. Math. 102 (1993) 20-78 MR1250465 
[27] M M Kapranov, V A Voevodsky, 2-categories and Zamolodchikov tetrahedra equations, from: "Algebraic groups and their generalizations: quantum and infinitedimensional methods (University Park, PA, 1991)", Proc. Sympos. Pure Math. 56, Amer. Math. Soc. (1994) 177-259 MR1278735

[28] S Lack, ICONS arXiv:0711.4657

[29] S Lack, S Paoli, 2-nerves for bicategories, K-Theory 38 (2008) 153-175 MR2366560

[30] S Mac Lane, Categories for the working mathematician, second edition, Graduate Texts in Math. 5, Springer, New York (1998) MR1712872

[31] JP May, Simplicial objects in algebraic topology, Van Nostrand Math. Studies 11, D Van Nostrand Co., Princeton-Toronto-London (1967) MR0222892

[32] J P May, The geometry of iterated loop spaces, Lectures Notes in Math. 271, Springer, Berlin (1972) MR0420610

[33] J P May, Pairings of categories and spectra, J. Pure Appl. Algebra 19 (1980) 299-346 MR593258

[34] I Moerdijk, J-A Svensson, Algebraic classification of equivariant homotopy 2-types. I, J. Pure Appl. Algebra 89 (1993) 187-216 MR1239560

[35] D Quillen, Higher algebraic $K$-theory. I, from: "Algebraic $K$-theory, I: Higher $K$ theories (Proc. Conf., Battelle Memorial Inst., Seattle, Wash., 1972)", Lecture Notes in Math. 341, Springer, Berlin (1973) 85-147 MR0338129

[36] G Segal, Classifying spaces and spectral sequences, Inst. Hautes Études Sci. Publ. Math. (1968) 105-112 MR0232393

[37] G Segal, Categories and cohomology theories, Topology 13 (1974) 293-312 MR0353298

[38] C Simson, A closed model structure for n-categories, internal hom, $n$-staks and generalized Seifert-Van Kampen arXiv:9704.5006

[39] R Street, Two constructions on lax functors, Cahiers Topologie Géom. Différentielle 13 (1972) 217-264 MR0347936

[40] R Street, The algebra of oriented simplexes, J. Pure Appl. Algebra 49 (1987) 283-335 MR920944

[41] R Street, Categorical structures, from: "Handbook of algebra, Vol. 1", North-Holland, Amsterdam (1996) 529-577 MR1421811

[42] Z Tamsamani, Sur des notions de n-catégorie et $n$-groupoïde non strictes via des ensembles multi-simpliciaux, $K$-Theory 16 (1999) 51-99 MR1673923

[43] R W Thomason, Homotopy colimits in the category of small categories, Math. Proc. Cambridge Philos. Soc. 85 (1979) 91-109 MR510404 
[44] U Tillmann, Discrete models for the category of Riemann surfaces, Math. Proc. Cambridge Philos. Soc. 121 (1997) 39-49 MR1418359

[45] U Tillmann, On the homotopy of the stable mapping class group, Invent. Math. 130 (1997) 257-275 MR1474157

[46] K Worytkiewicz, K Hess, P E Parent, A Tonks, A model structure à la Thomason on 2-Cat, J. Pure Appl. Algebra 208 (2007) 205-236 MR2269840

Departamento de Álgebra, Facultad de Ciencias, Universidad de Granada 18071 Granada, Spain

mcarrasc@ugr.es, acegarra@ugr.es, agarzon@ugr.es

www.ugr.es/local/acegarra, www.ugr.es/local/agarzon

Received: 30 March 2009 